\title{
A posturographic procedure assessing balance disorders in Parkinson's disease: a systematic review
}

This article was published in the following Dove Press journal:

Clinical Interventions in Aging

\section{Anna Kamieniarz \\ Justyna Michalska \\ Anna Brachman \\ Michał Pawłowski \\ Kajetan J Słomka \\ Grzegorz Juras}

Department of Human Motor Behavior, The Jerzy Kukuczka Academy of Physical Education in Katowice, Katowice, Poland
Correspondence: Anna Kamieniarz The Jerzy Kukuczka Academy of Physical Education in Katowice, 72a Mikolowska Street, 40-065 Katowice, Poland Tel +4832 2075160

Email a.kamieniarz@awf.katowice.pl Department of Human Motor Behavior,

\begin{abstract}
Postural instability is common in Parkinson's disease (PD), often contributing to falls, injuries, and reduced mobility. In the clinical setting, balance disorder is commonly diagnosed using clinical tests and balance scales, but it is suggested that the most sensitive measurement is the force platform. The aim of this systematic review was to summarize the methods and various posturographic procedures used to assess the body balance and gait in PD. A systematic review was conducted of papers published from 2000 to 2017. Databases searched were PubMed and EBSCO. Studies must have involved patients with PD, used force platform or motion analysis system as a measurement tool, and described posturographic procedure. The Physiotherapy Evidence Database (PEDro) scale was used to assess the methodological quality of the included studies. A total of 32 studies met the inclusion criteria. The PEDro scores ranged from 5 to 7 points. The analysis of the objective methods assessing balance disorders revealed a large discrepancy in the duration and procedures of measurements. The number of repetitions of each trial fluctuated between 1 and 8 , and the duration of a single trial ranged from 10 to 60 seconds. Overall, there are many scales and tests used to assess the balance disorders and disabilities of people with PD. Although in many included studies the authors have used posturography as a method to evaluate the postural instability of PD patients, the results are contradictory. To solve this issue, it is indicated to establish a "gold standard" of procedures of measures of balance.
\end{abstract}

Keywords: Parkinson's disease, posturography, balance disorders, postural instability

\section{Introduction}

Maintenance of body balance is important for daily life. Balance is the ability of the human body to maintain the position of its center of gravity (COG) within the area of its base of support (BOS), and balance requires a continuous feedback system that processes visual, vestibular, and somatosensory inputs and executes neuromuscular actions. ${ }^{1,2}$ However, Hof ${ }^{3}$ introduced the concept of the "extrapolated center of mass" $\mathrm{XcoM}$, and the authors proposed the new definition of human balance: the XcoM must remain within the boundaries of the BOS.

Parkinson's disease (PD) is a chronic and progressive degenerative disorder of the central nervous system, which is characterized by resting tremor, rigidity, bradykinesia, and postural instability. PD affects all age groups, but it is most commonly found in the elderly population. ${ }^{4}$

Postural instability is a common clinical problem in PD which often contributes to falls, injuries, and reduced mobility. Postural instability is due to a dysfunction of postural reflexes and is generally a manifestation of the late stages of the disease. ${ }^{5}$ 
A number of clinical tests and balance scales have been used to assess the postural stability, the risk of falling, and motor symptoms in PD. ${ }^{6}$ Despite widely used clinical tests, research suggests that some of the tests are not able to identify differences in postural instability and mobility between people with PD and age-matched healthy adults. ${ }^{7-9}$ Moreover, postural instability is diagnosed in stage 3 in Hoehn and Yahr (H\&Y) scale, ${ }^{10}$ but there are some evidences that the postural sway can be abnormal in the early stage of PD before the onset of clinical symptoms. ${ }^{11}$ This fact is extremely important, when one use existing methods to identify and quantify the balance disorders in PD patients who do not complain about problems with their postural instability. Nardone and Schieppati ${ }^{12}$ suggested posturography to be a useful technique of an early recognition and evaluation of balance dysfunction in PD. This is crucial in clinical practice. Also, with the use of posturography, one may gain a better understanding of the pathophysiological mechanisms of balance disorders in PD. ${ }^{13,14}$ Therefore, force plate posturography is a major tool to recognize the balance dysfunction in $\mathrm{PD}$, and it can provide a more objective and sensitive measure of postural instability. This notion is confirmed by the extensive literature on its use in PD. ${ }^{15-17}$ In addition, according to the Evidence-Based Medicine policy, the most valuable and reliable scientific evidence is based on an objective assessment, ${ }^{18}$ and the posturography is the one of the such methods.

The main purpose of this study was to conduct a systematic review of the published literature on the different methods used to assess balance and gait in individuals with PD.
In addition, this systematic review summarized various posturographic procedures and standard measures of postural sway variability used in studies on balance disorders in PD.

\section{Methods}

\section{Search strategy}

A comprehensive systematic literature search was performed using the following databases: PubMed and EBSCO. We mainly selected articles that were published between 2000 and 2017. Combinations of the following key terms were used for each database: "body balance” OR "postural control” OR "posturography" OR "gait initiation" OR "crossing obstacle" AND "Parkinson's disease". The search was limited to articles published in English and full-text original articles.

\section{Study selection}

Early screening of the articles based on titles and abstracts was performed by two authors (AB and MP). The full-text articles were independently assessed by two authors (AK and JM) using the following study inclusion criteria: 1) the participants of the studies had to suffer from PD, 2) the outcome measures had to be a force platform and/or a video camera and/or clinical tests, and 3) the posturographic procedure was described. Studies were excluded if 1) there was no control group, 2) the Physiotherapy Evidence Database (PEDro) scale was lower than 4, and 3) there was a treatment intervention. The results of the study selection procedure are summarized in the flowchart. In total, 32 studies met the inclusion criteria for the review (Figure 1).

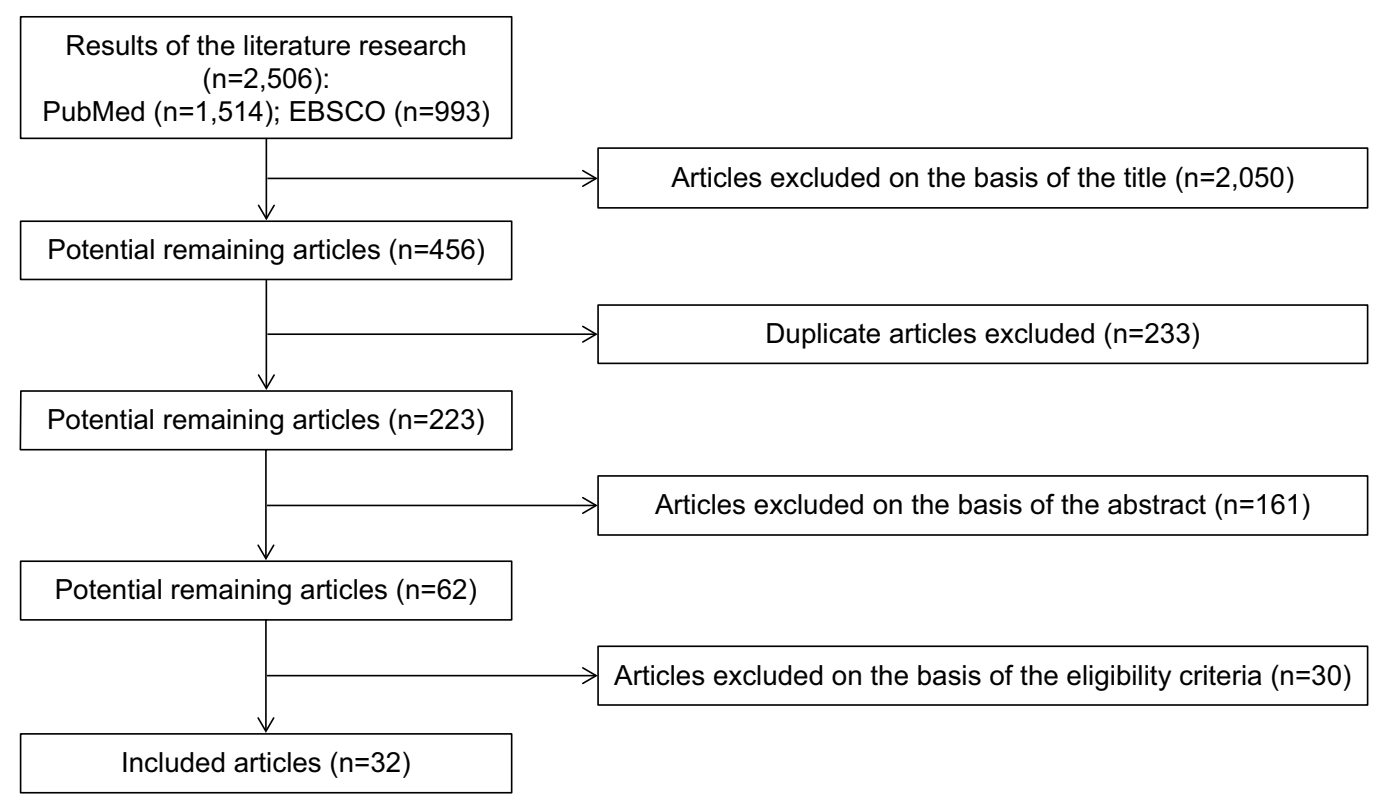

Figure I Flowchart for the study search and selection. 


\section{Methodological quality}

The quality of the included articles was assessed by three reviewers (AK, JM, and $\mathrm{AB}$ ) using the standardized and validated PEDro tool. The PEDro scale is an 11-item scale that has been previously used in systematic reviews. ${ }^{19,20}$ Any disagreements were resolved via discussion or consultation with the fourth author (KJS).

\section{Data extraction}

Two reviewers (AK and MP) extracted the following information independently from the included studies: study design, assessment protocols, outcome measures, and study conclusion. The data were collated and organized in Tables 1 and 2 .

\section{Results}

\section{Data synthesis}

The initial search retrieved a total of 2,506 articles from the databases. After exclusion on the basis of the title and removal of duplicate articles, 223 potential articles were identified. After the abstract review, 62 full-text articles were assessed for the eligibility criteria. Finally, 32 articles met all the inclusion criteria and were included in our systematic review. Figure 1 presents a flowchart of the literature search process.

\section{Participants' characteristics}

The total pooled sample size of all of the included studies was 791 participants, including 383 males and 228 females. The mean age of the subjects ranged between 40 and 90 years among the included studies. A summary of the included studies is presented in Table 3 .

\section{Quality assessment}

All of the included studies had a quality score ranging from 5 to 7 points. Of the included studies, 8 were graded as fair and 24 were graded as good. Table 3 illustrates the PEDro points of all the included studies.

\section{Assessment of balance in PD}

Subjective assessment of balance and motor abilities In our review, we found that the H\&Y scale was commonly used to assess disease progression from stage 0 (absence of disease symptoms) to stage 5 (wheelchair mobility). ${ }^{10}$ In almost all of the studies, individuals with PD had H\&Y stage I-III ( $n=27)$, suggesting a mild to moderate stage of the disease. In addition, the most widely used $(\mathrm{n}=24)$ scale was the Unified Parkinson's Disease Rating Scale (UPDRS), ${ }^{21}$

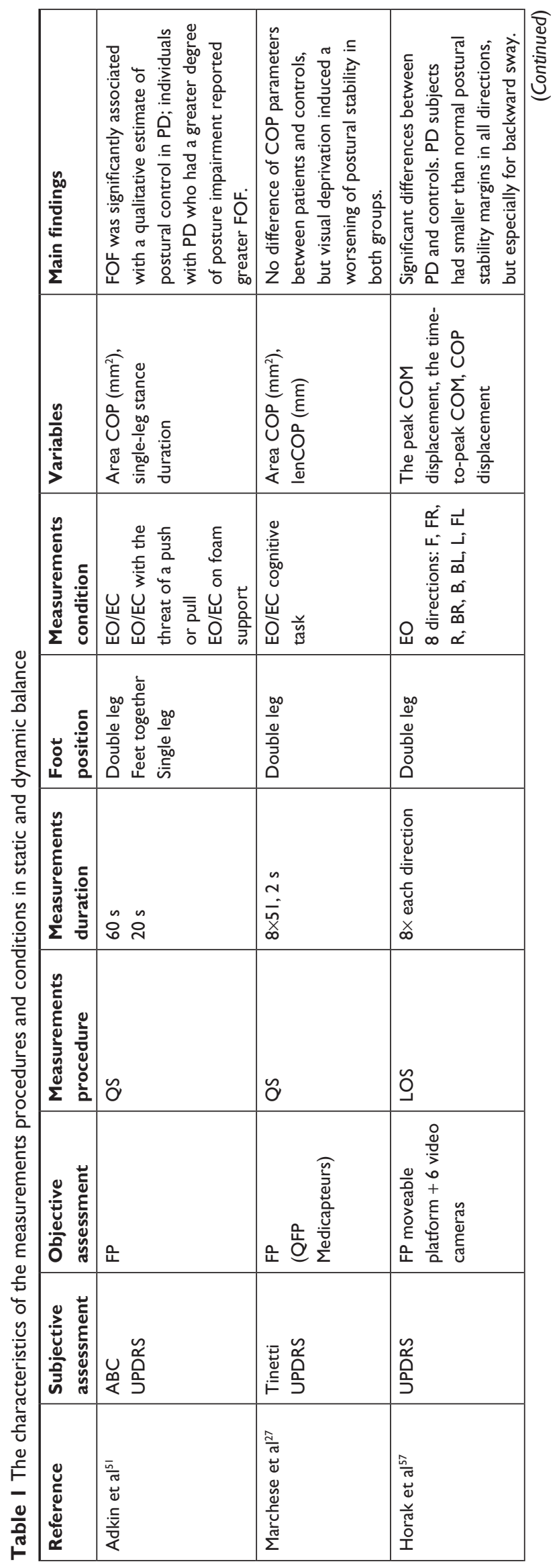




\begin{tabular}{|c|c|c|c|c|c|c|}
\hline 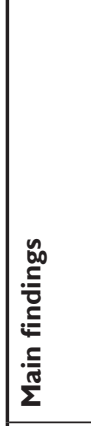 & 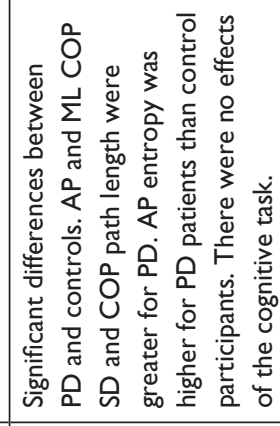 & 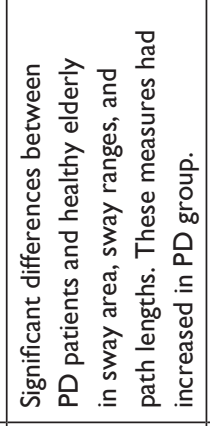 & 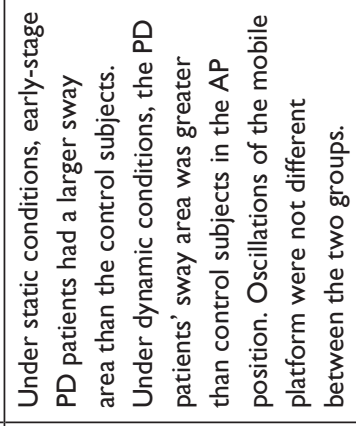 & 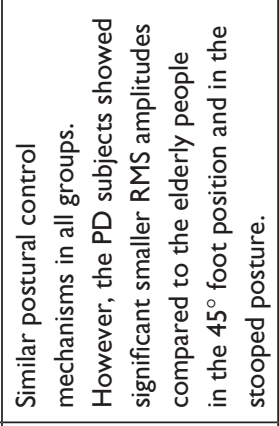 & 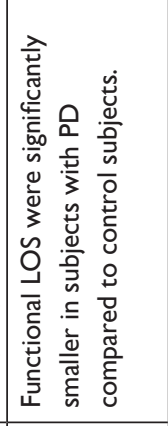 & 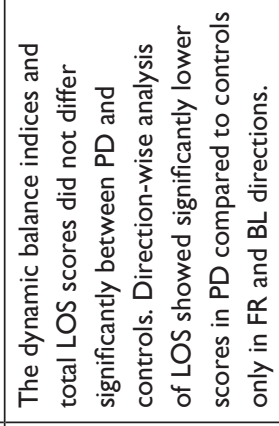 \\
\hline$\frac{y}{\frac{y}{0}}$ & 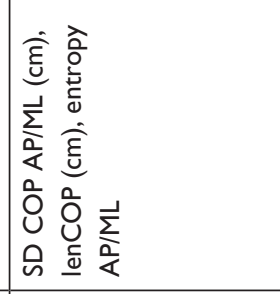 & 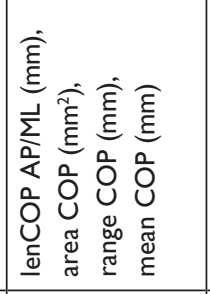 & 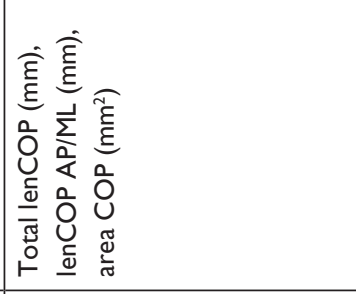 & 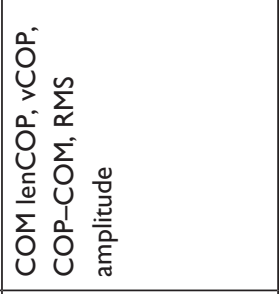 & 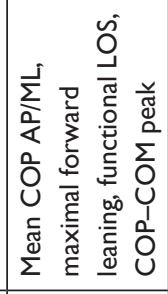 & 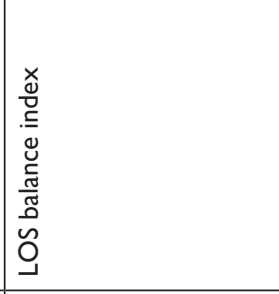 \\
\hline 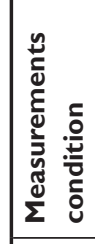 & 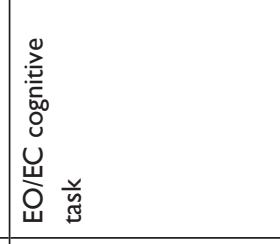 & \begin{tabular}{|l}
$\mathrm{u}$ \\
엉 \\
\end{tabular} & $\begin{array}{l}\text { u } \\
\text { Oे } \\
\end{array}$ & 只 & 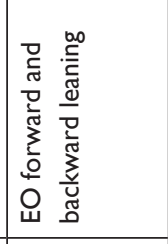 & 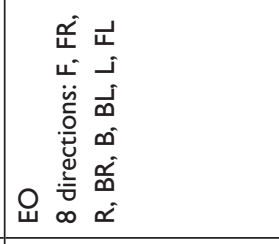 \\
\hline 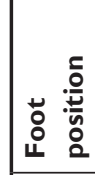 & 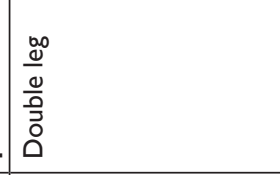 & \begin{tabular}{|l|}
$\frac{00}{\frac{0}{0}}$ \\
$\frac{0}{0}$ \\
$\frac{0}{0}$ \\
0 \\
0
\end{tabular} & \begin{tabular}{|l}
$\frac{0}{0}$ \\
$\frac{0}{0}$ \\
$\frac{0}{0}$ \\
0 \\
0 \\
\end{tabular} & 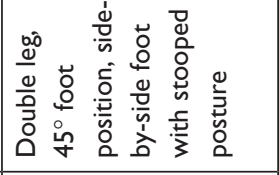 & 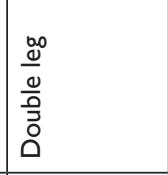 & 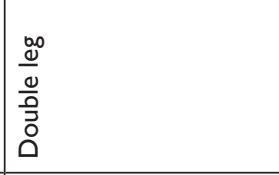 \\
\hline 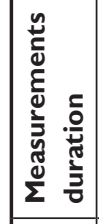 & 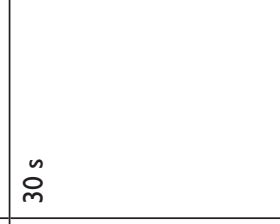 & 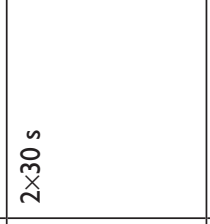 & فุ & \begin{tabular}{|l}
$n$ \\
0 \\
$x$ \\
$n$ \\
\end{tabular} & 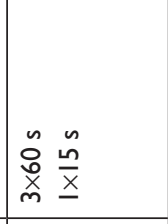 & 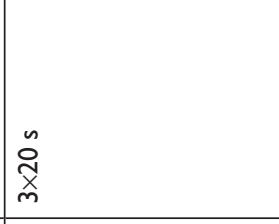 \\
\hline 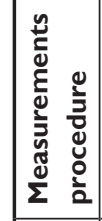 & \% & ŏ & $\tilde{\partial}$ & $\breve{~}$ & 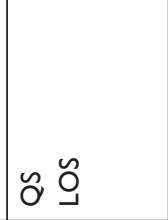 & ô \\
\hline 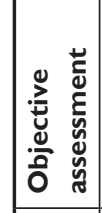 & 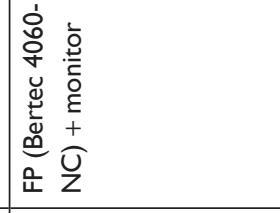 & 운 产 & 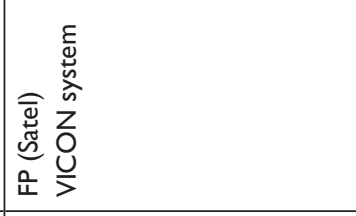 & 은 & 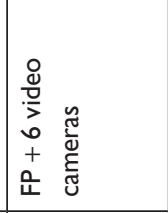 & 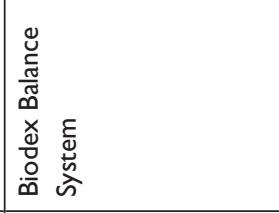 \\
\hline 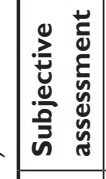 & 1 & 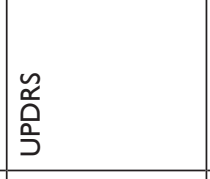 & 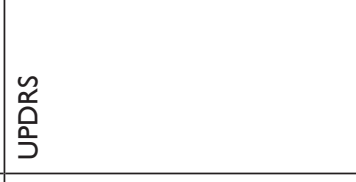 & 1 & 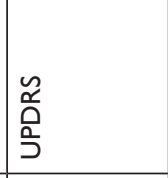 & 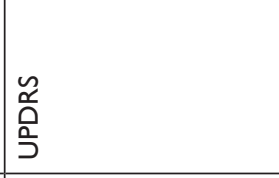 \\
\hline 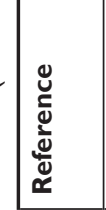 & 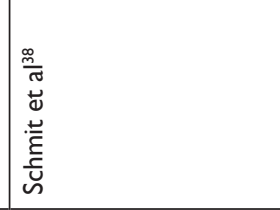 & 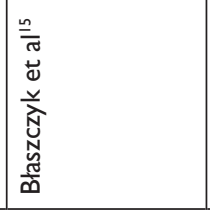 & 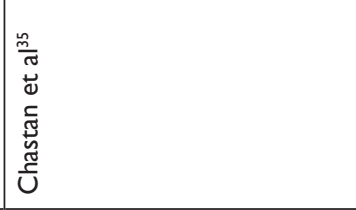 & 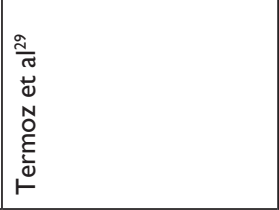 & 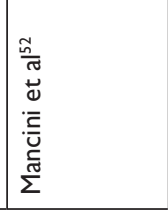 & 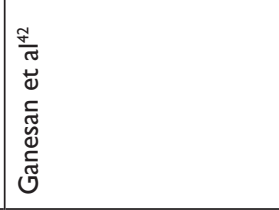 \\
\hline
\end{tabular}




\begin{tabular}{|c|c|c|c|c|c|c|}
\hline 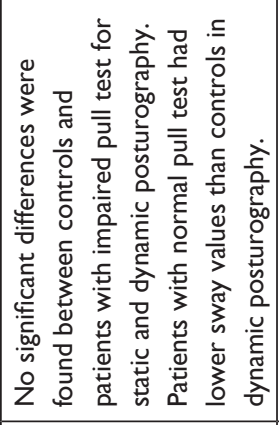 & 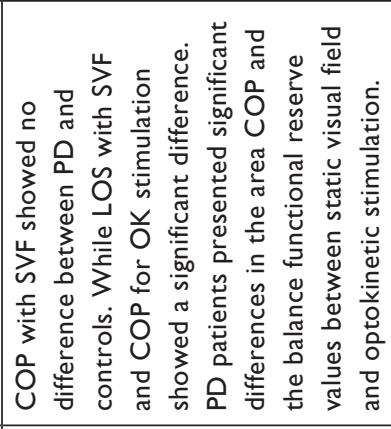 & 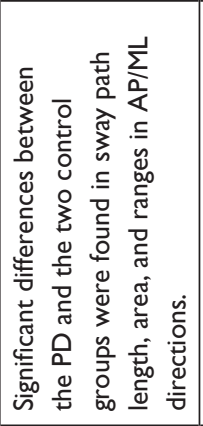 & 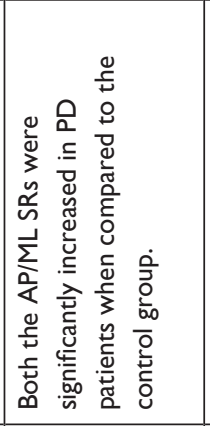 & 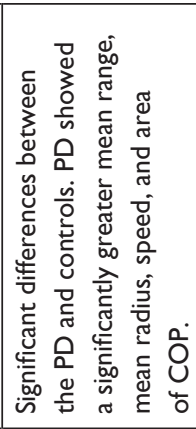 & 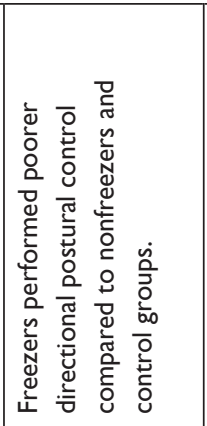 & 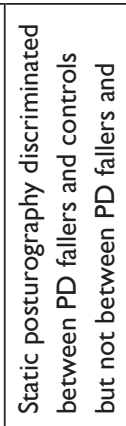 \\
\hline 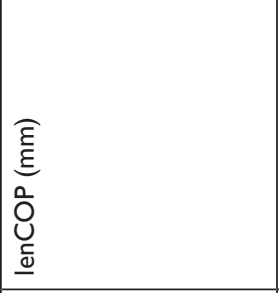 & 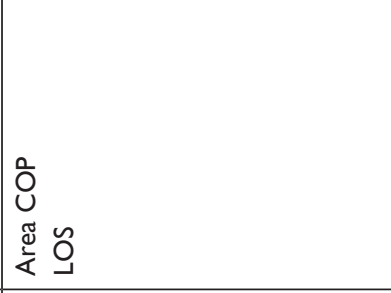 & 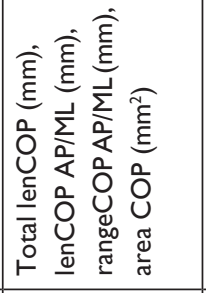 & 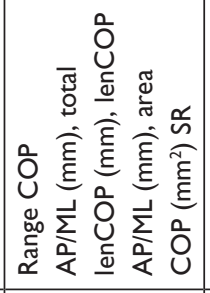 & 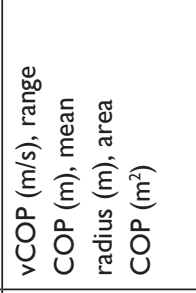 & 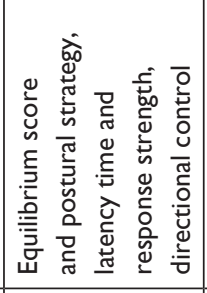 & 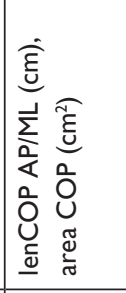 \\
\hline 只 & 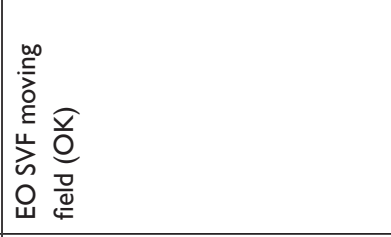 & 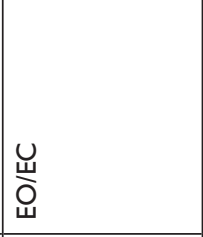 & $\begin{array}{l}u \\
\text { 岁 } \\
\text { u }\end{array}$ & 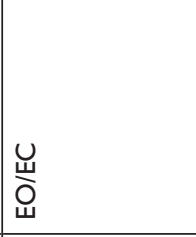 & 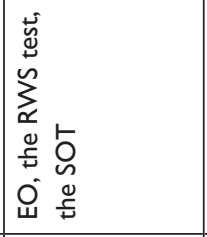 & \begin{tabular}{|l}
$u$ \\
岁 \\
\end{tabular} \\
\hline 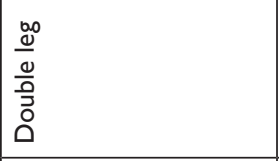 & 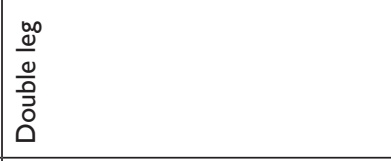 & \begin{tabular}{|l}
$\frac{\infty}{0}$ \\
$\frac{0}{0}$ \\
$\frac{0}{0}$ \\
$\overline{0}$ \\
0 \\
\end{tabular} & \begin{tabular}{|l}
$\frac{80}{0}$ \\
$\frac{0}{0}$ \\
0 \\
0 \\
0 \\
\end{tabular} & 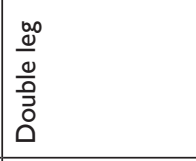 & 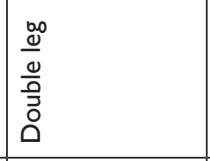 & \begin{tabular}{|l}
$\frac{\infty}{0}$ \\
$\frac{0}{0}$ \\
$\frac{0}{0}$ \\
0 \\
0
\end{tabular} \\
\hline $\begin{array}{l}n \\
8 \\
0\end{array}$ & 孚 & $\begin{array}{l}n \\
0 \\
\tilde{\tilde{m}} \\
\tilde{m}\end{array}$ & 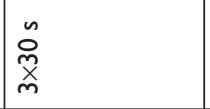 & $\begin{array}{l}n \\
\stackrel{\tilde{\tilde{N}}}{x} \\
\text { N }\end{array}$ & $\begin{array}{l}n \\
\tilde{\tilde{x}} \\
\tilde{x}\end{array}$ & 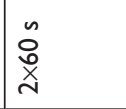 \\
\hline$\tilde{\partial}$ & Oิ & $\tilde{O}$ & $\widetilde{O}$ & $\tilde{O}$ & oิ & $\tilde{O}$ \\
\hline 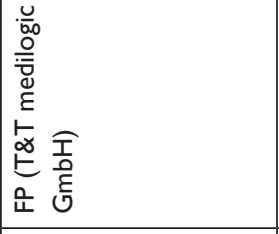 & 这 & 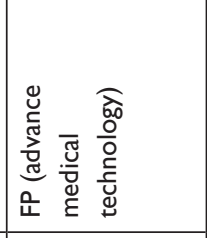 & 운 & 遂 & 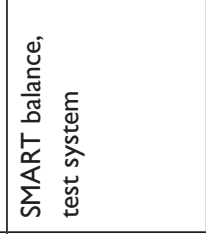 & 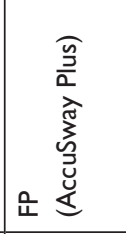 \\
\hline 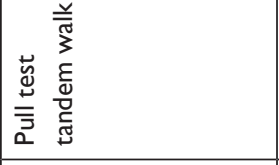 & 1 & 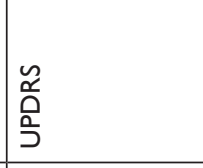 & 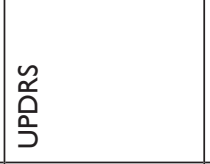 & 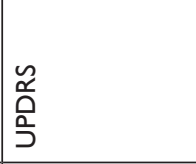 & 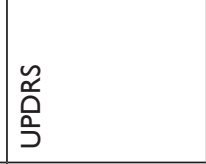 & 㟧 \\
\hline 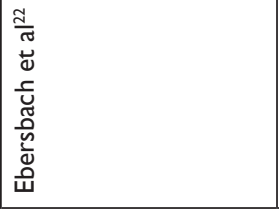 & 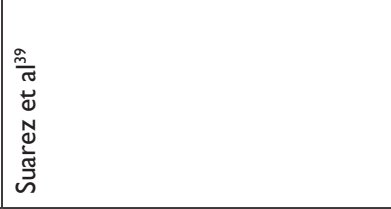 & 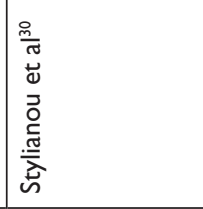 & 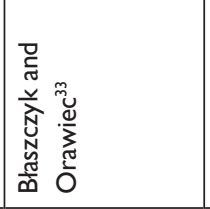 & 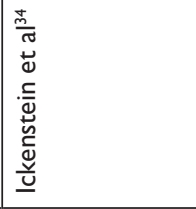 & 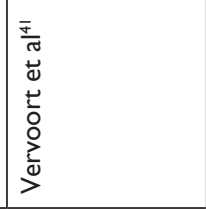 & 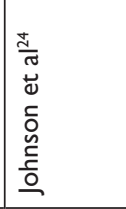 \\
\hline
\end{tabular}




\begin{tabular}{|c|c|c|c|c|c|}
\hline 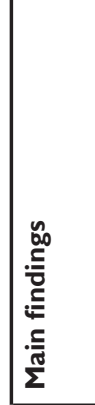 & 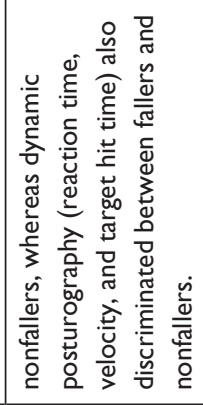 & 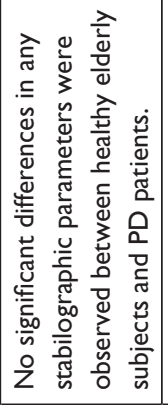 & 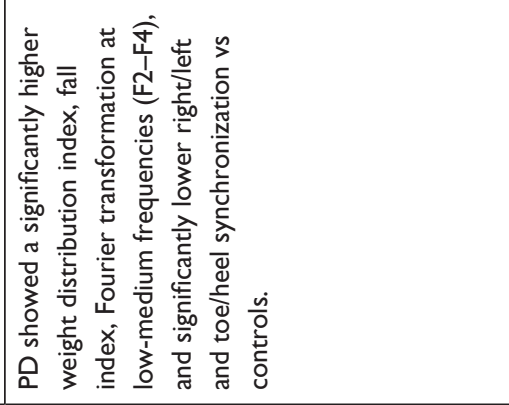 & 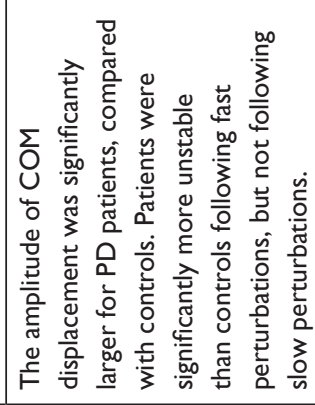 & 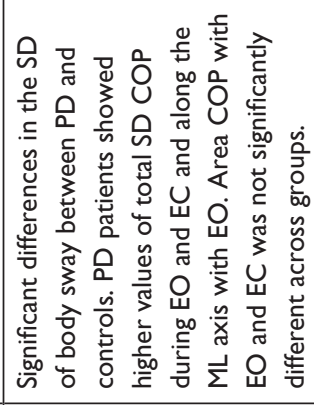 \\
\hline $\begin{array}{l}\frac{y}{0} \\
\frac{0 \pi}{\frac{\pi}{n}} \\
>\end{array}$ & & 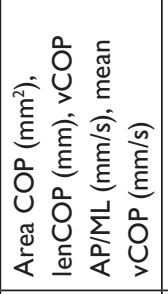 & 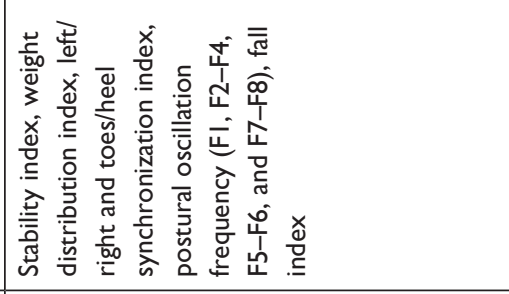 & 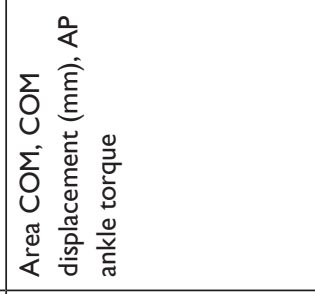 & 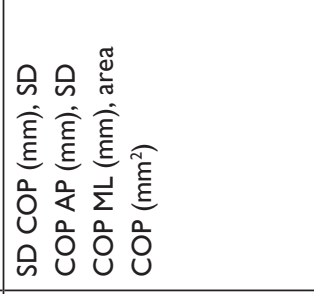 \\
\hline 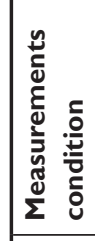 & & 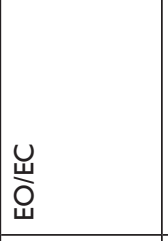 & 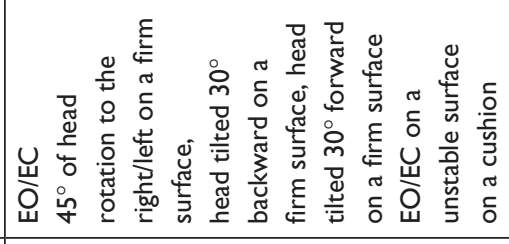 & 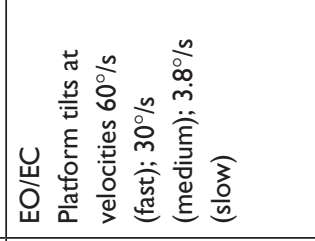 & 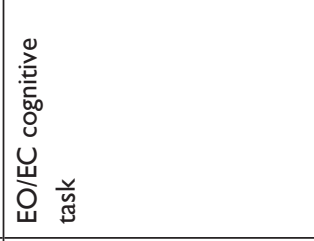 \\
\hline 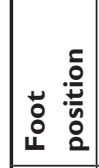 & & 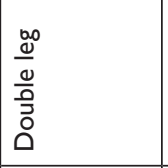 & \begin{tabular}{|l}
$\frac{0}{0}$ \\
$\frac{0}{0}$ \\
$\frac{0}{0}$ \\
0 \\
0 \\
0
\end{tabular} & \begin{tabular}{|l|}
$\frac{\infty}{0}$ \\
$\frac{0}{0}$ \\
$\frac{0}{0}$ \\
$\overline{0}$ \\
0 \\
\end{tabular} & 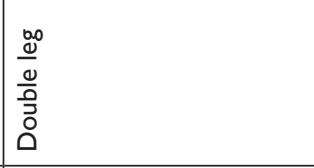 \\
\hline 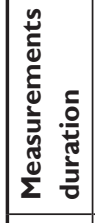 & & $\underset{\sim}{\tilde{N}}$ & $\underset{\tilde{m}}{\tilde{m}}$ & 爻 & 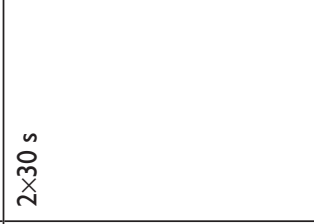 \\
\hline 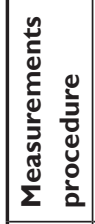 & & $\tilde{O}$ & ŏ & ô & ô \\
\hline 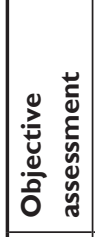 & & 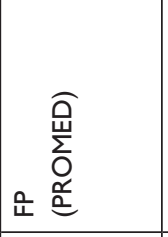 & 욘 & 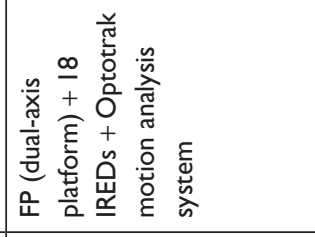 & 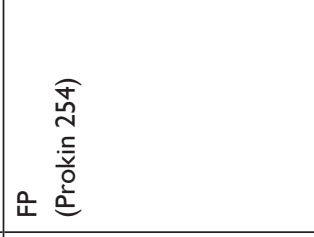 \\
\hline 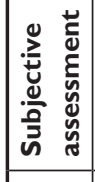 & 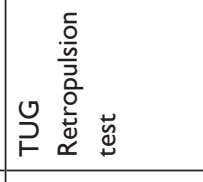 & 1 & 1 & 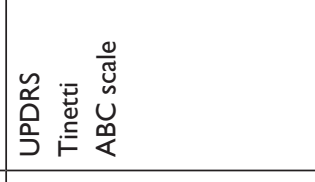 & 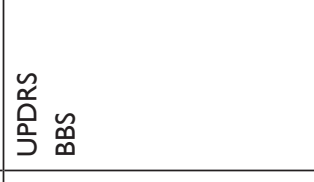 \\
\hline 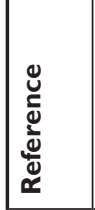 & & 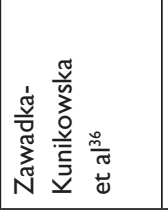 & 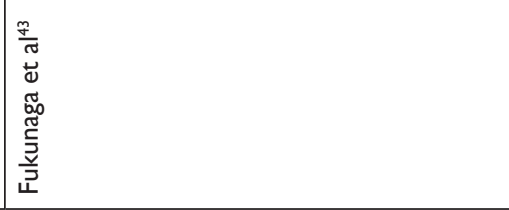 & 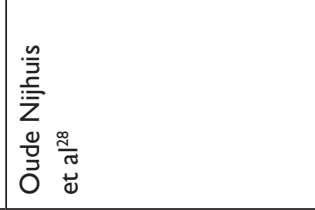 & 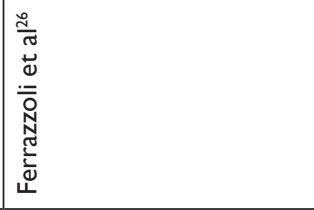 \\
\hline
\end{tabular}




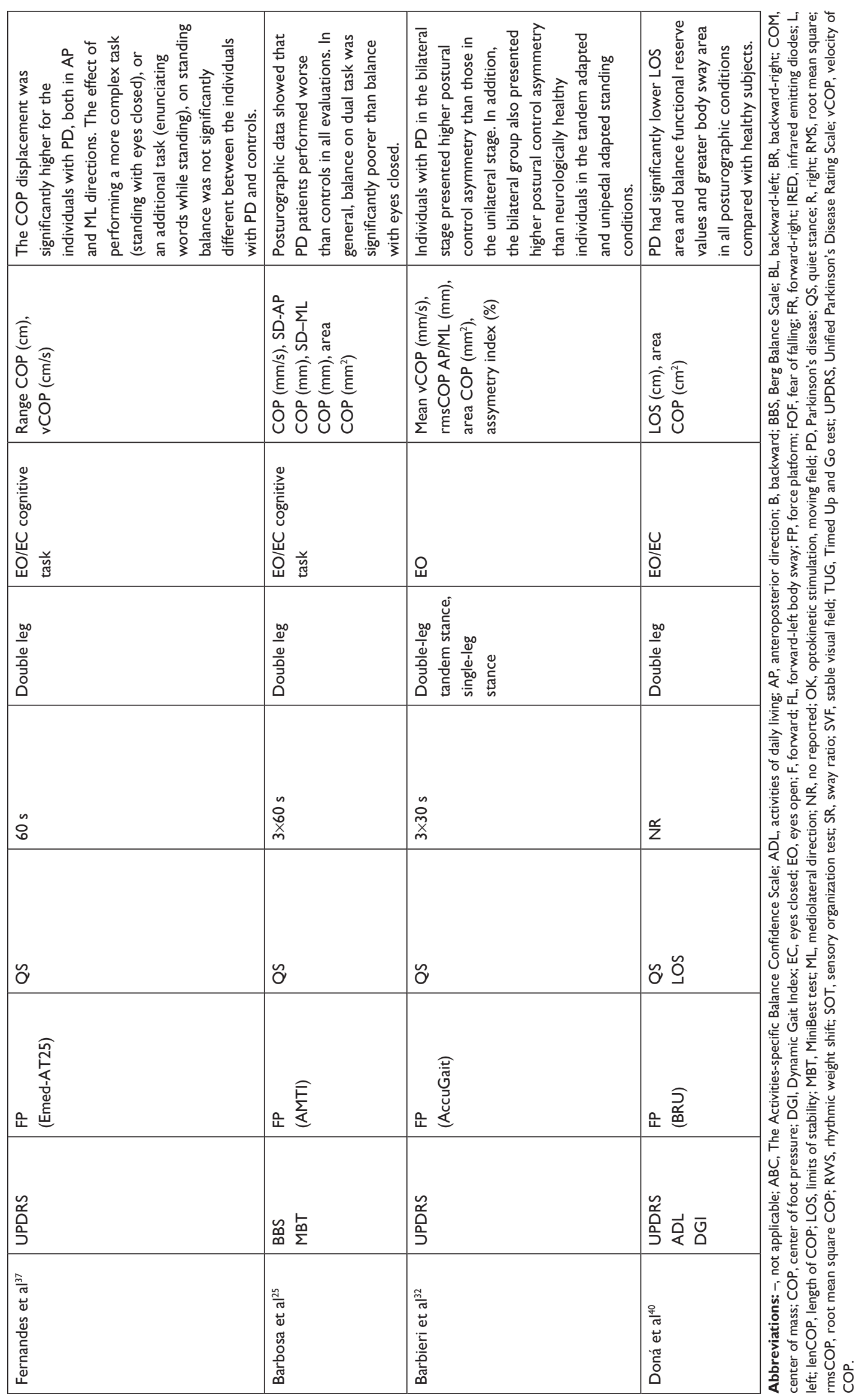




\begin{tabular}{|c|c|c|c|c|c|}
\hline 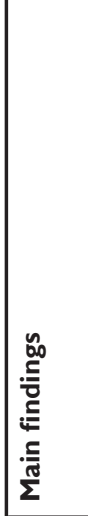 & 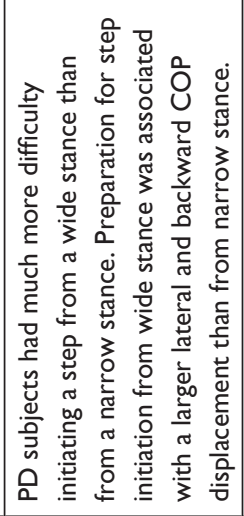 & 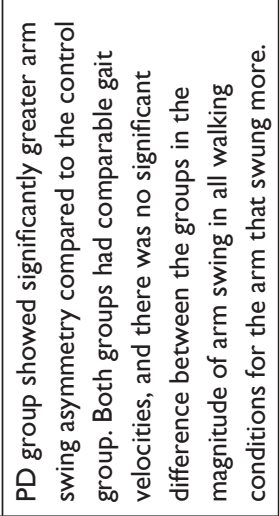 & 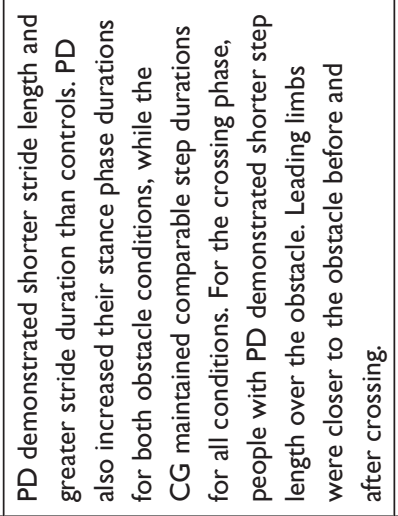 & 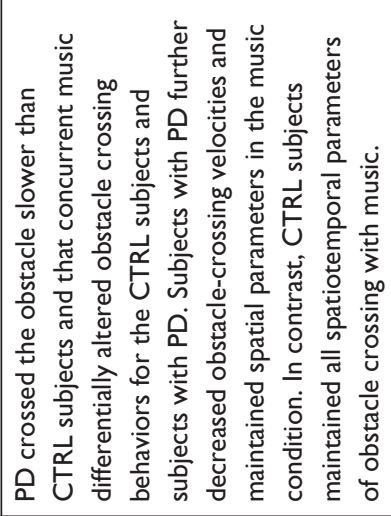 & 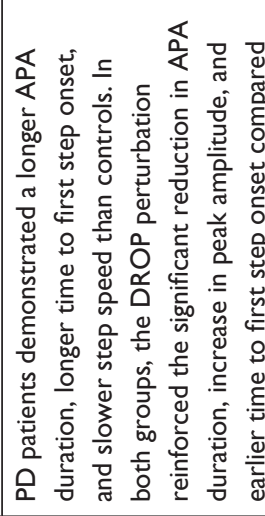 \\
\hline $\begin{array}{l}\frac{\tilde{y}}{0} \\
\frac{\sqrt{\pi}}{\frac{\pi}{\pi}} \\
>\end{array}$ & 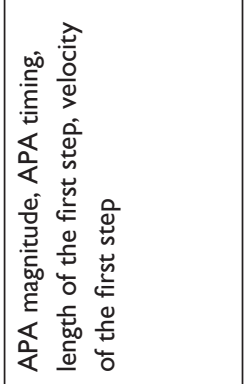 & 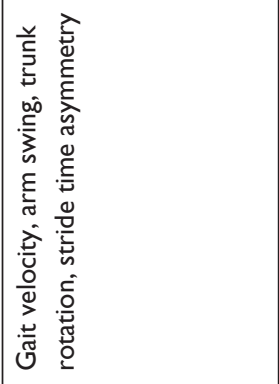 & 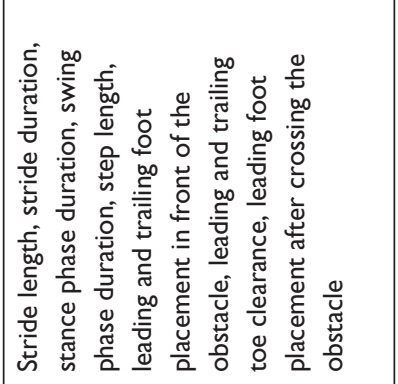 & 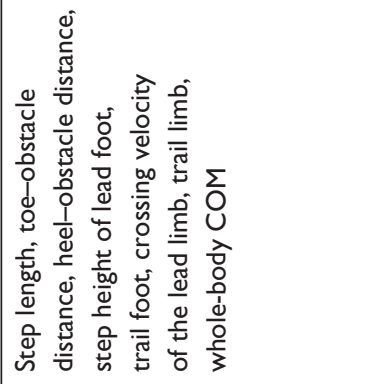 & 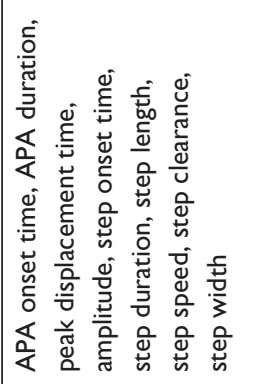 \\
\hline 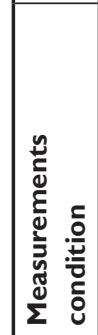 & 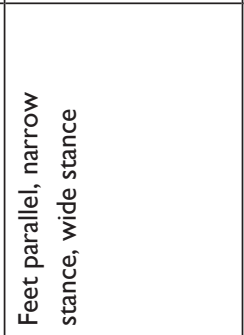 & 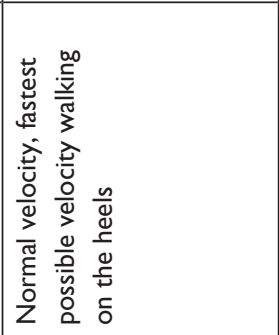 & 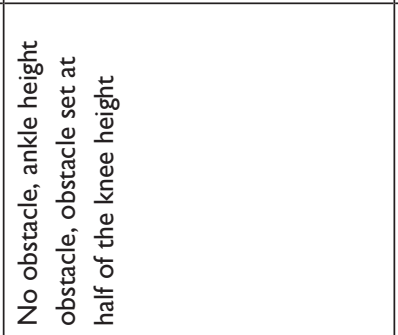 & 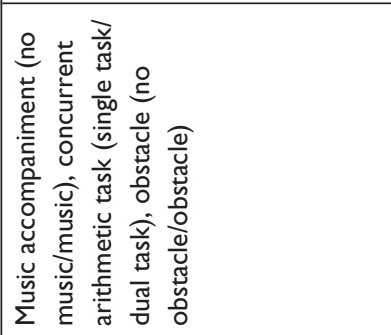 & 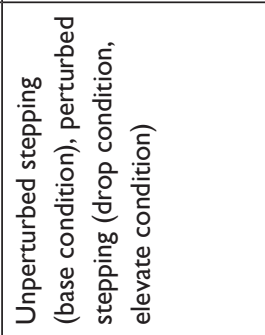 \\
\hline 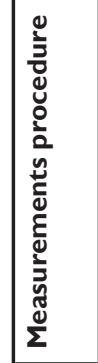 & 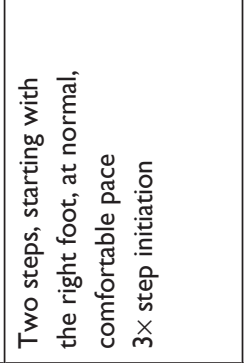 & 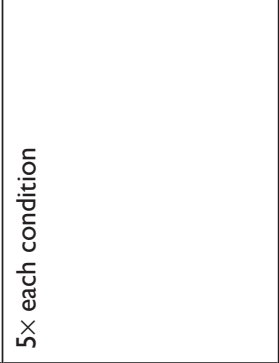 & 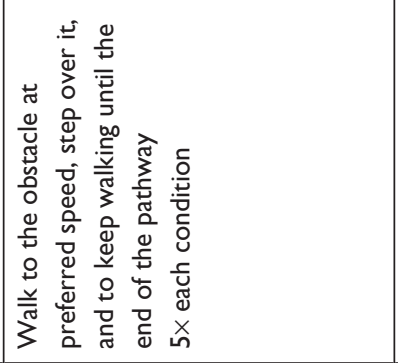 & 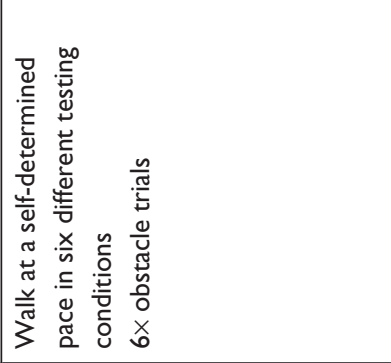 & 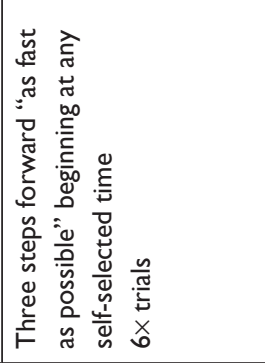 \\
\hline 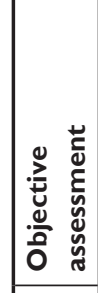 & 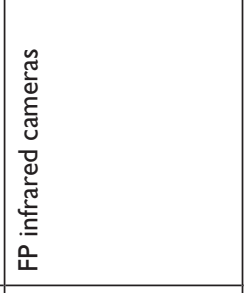 & 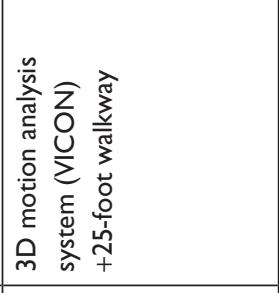 & 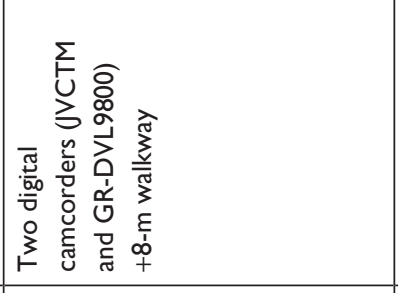 & 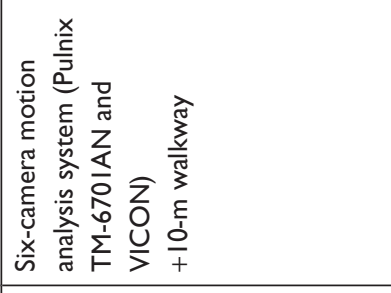 & 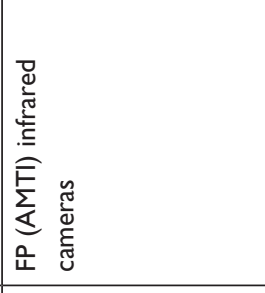 \\
\hline 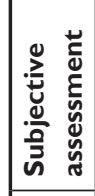 & 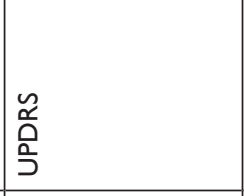 & 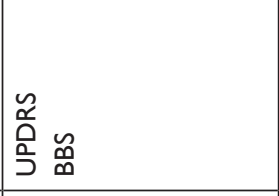 & 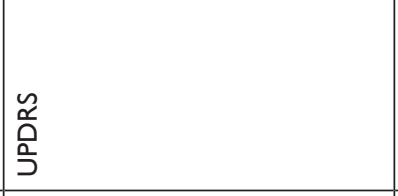 & 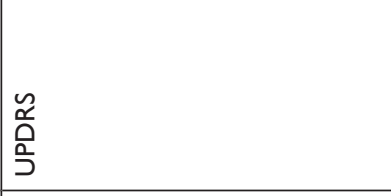 & $\begin{array}{l}\mathscr{\varkappa} \\
0 \\
\frac{0}{\partial}\end{array}$ \\
\hline 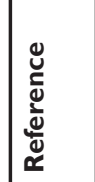 & 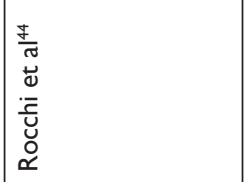 & 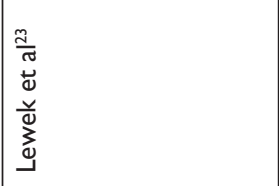 & 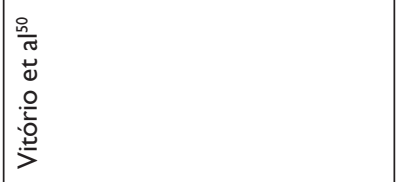 & 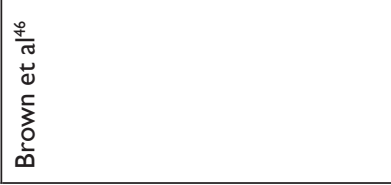 & 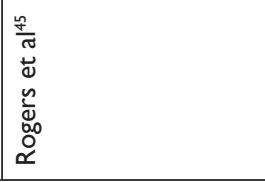 \\
\hline
\end{tabular}




\begin{tabular}{|c|c|c|c|}
\hline \multirow[t]{3}{*}{ 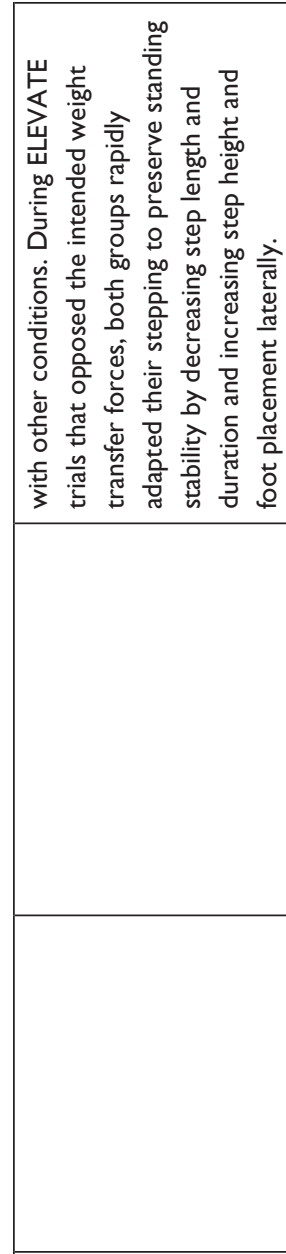 } & 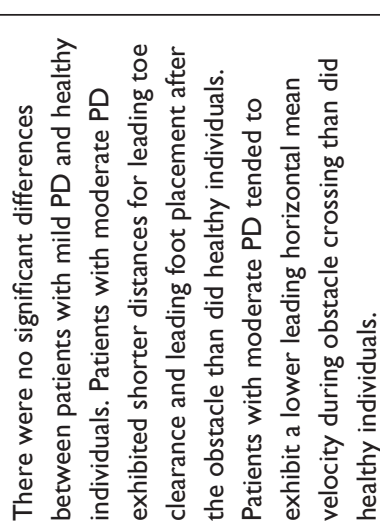 & 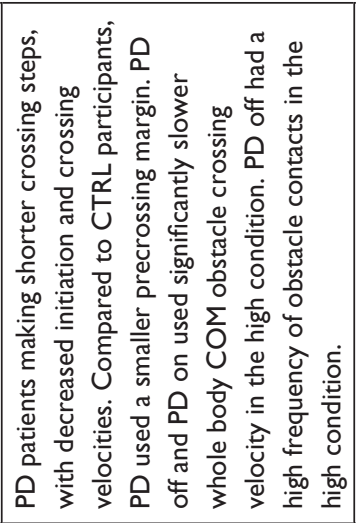 & 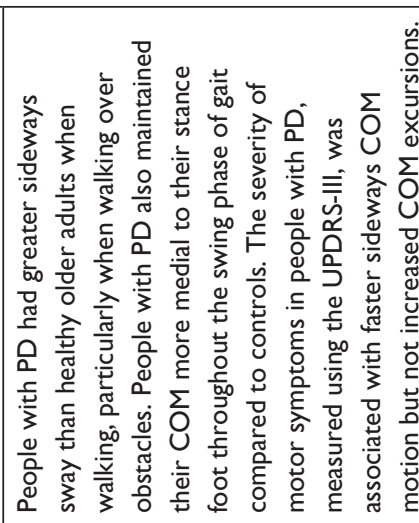 \\
\hline & 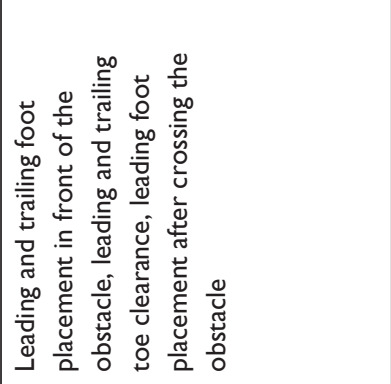 & 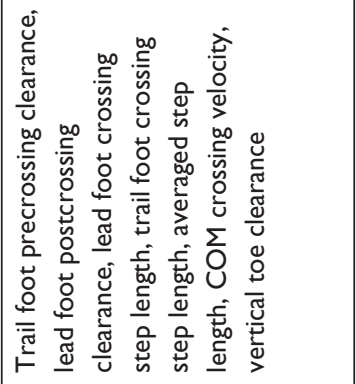 & 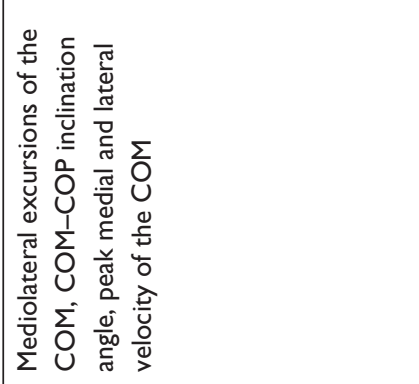 \\
\hline & 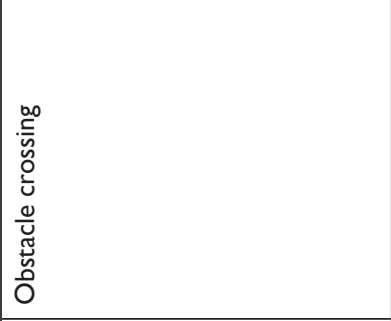 & 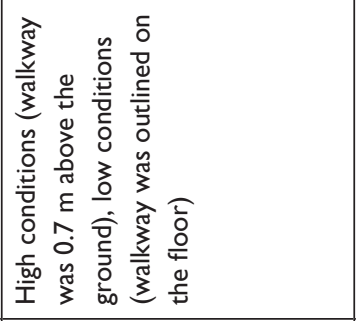 & 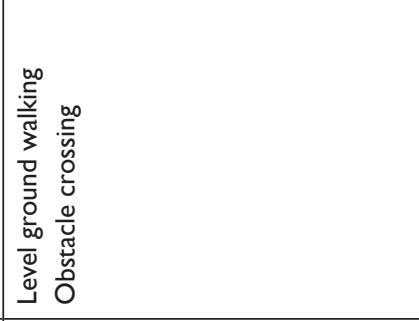 \\
\hline & 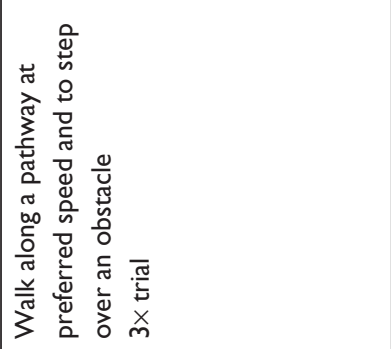 & 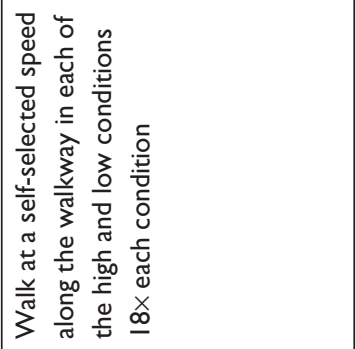 & 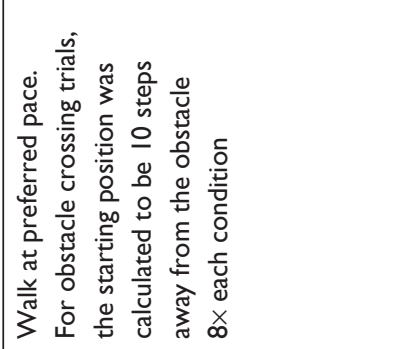 \\
\hline & 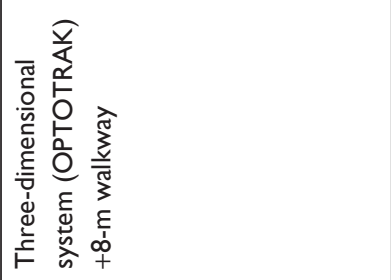 & 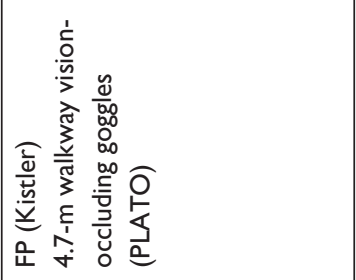 & 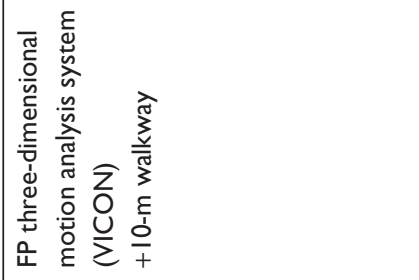 \\
\hline & 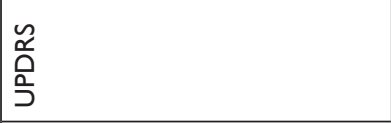 & 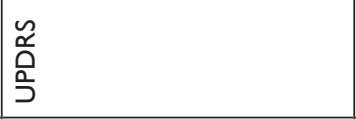 & $\begin{array}{l}\mathscr{y} \\
\text { مै } \\
\frac{0}{J}\end{array}$ \\
\hline & 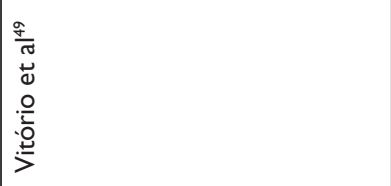 & 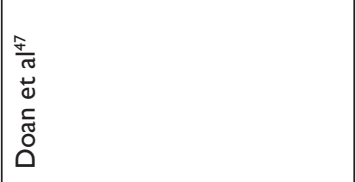 & 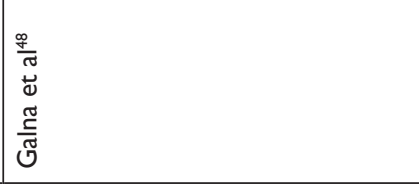 \\
\hline
\end{tabular}


Table 3 A summary of the included studies - group characteristics

\begin{tabular}{|c|c|c|c|c|c|c|c|c|}
\hline \multirow[t]{2}{*}{ Reference } & \multicolumn{5}{|l|}{ PD } & \multicolumn{2}{|l|}{ Control } & \multirow[t]{2}{*}{ PEDro } \\
\hline & $n(F / M)$ & $\begin{array}{l}\text { Age (years), } \\
\text { mean } \pm \text { SD }\end{array}$ & $\begin{array}{l}\text { H\&Y } \\
\text { scale }\end{array}$ & $\begin{array}{l}\text { Disease duration } \\
\text { (years), mean } \pm \text { SD }\end{array}$ & UPDRS & n (F/M) & $\begin{array}{l}\text { Age (years), } \\
\text { mean } \pm \text { SD }\end{array}$ & \\
\hline Adkin et $\mathrm{a}^{51}$ & $58(19 / 39)$ & $66.2 \pm 9.3$ & NR & $6.5 \pm 4.9$ & NR & $30(16 / 14)$ & $66.7 \pm 8.1$ & 6 \\
\hline Marchese et al ${ }^{27}$ & $24(8 / 16)$ & $66.4 \pm 7.9$ & II-III & $8.9 \pm 3.3$ & NR & $20(7 / 13)$ & $60.9 \pm 7.4$ & 5 \\
\hline Horak et $\mathrm{a}^{57}$ & 7 (NR) & $67.0 \pm 2.0$ & III-IV & $17.6 \pm 5.0$ & $56.5 \pm 6.0$ & 7 (NR) & $66.3 \pm 2.2$ & 6 \\
\hline Schmit et al ${ }^{38}$ & $6(4 / 2)$ & $70.8 \pm 15.9$ & III & $6.2 \pm 1.1$ & NR & $6(4 / 2)$ & $70 .|7 \pm 4.7|$ & 6 \\
\hline Błaszczyk et al ${ }^{15}$ & $55(20 / 35)$ & $64.6 \pm 8.9$ & I-III & $5.5 \pm 4.4$ & NR & $55(20 / 35)$ & $64.3 \pm 7.9$ & 6 \\
\hline Chastan et $\mathrm{al}^{35}$ & $9(4 / 5)$ & $61.7 \pm 8.4$ & 1 & $3.2 \pm 2.0$ & $10.3 \pm 2.0$ & $18(8 / 10)$ & $60.6 \pm 7.4$ & 6 \\
\hline Termoz et $\mathrm{al}^{29}$ & 10 (NR) & 60.2 & II-III & $5.0 \pm 3.3$ & NR & 10 (NR) & 60.4 & 5 \\
\hline Mancini et $\mathrm{a}^{52}$ & $13(6 / 7)$ & $60 . \pm 8.5$ & I-II & $12.5 \pm 5.1$ & $28.5 \pm 14.5$ & 10 (NR) & $64.9 \pm 8$ & 6 \\
\hline Ganesan et $\mathrm{al}^{42}$ & 20 (NR) & $58.3 \pm 8.7$ & II & $3.6 \pm 2.0$ & $28.3 \pm 5.0$ & 20 (NR) & $57.9 \pm 8.5$ & 5 \\
\hline Ebersbach et a ${ }^{22}$ & $58(23 / 35)$ & $65.9 \pm 7.8$ & I-III & $9.5 \pm 6.3$ & $16.1 \pm 12.0$ & $29(15 / 14)$ & $66.8 \pm 6.4$ & 6 \\
\hline Suarez et $\mathrm{al}^{39}$ & 24 (NR) & $66.5 \pm 8.5$ & I-II & NR & NR & 19 (NR) & $62.3 \pm 12.7$ & 5 \\
\hline Stylianou et $\mathrm{al}^{30}$ & 19 (NR) & $65.0 \pm 9.7$ & I-III & $5.2 \pm 4.7$ & $21.3 \pm 8.5$ & I4 (NR) & $67.8 \pm 9.6$ & 5 \\
\hline $\begin{array}{l}\text { Błaszczyk and } \\
\text { Orawiec }^{33}\end{array}$ & $55(20 / 35)$ & $64.6 \pm 8.9$ & I-III & $5.4 \pm 4.4$ & $23.3 \pm 12.1$ & $55(20 / 35)$ & $64.3 \pm 7.9$ & 6 \\
\hline Ickenstein et a ${ }^{34}$ & $12(6 / 6)$ & $71.9 \pm 5.8$ & I-III & NR & NR & $10(6 / 4)$ & $72.0 \pm 6.9$ & 6 \\
\hline Vervoort et $\mathrm{a}^{41}$ & $19(2 / 17)$ & $58-75$ & II-IV & $9.0 \pm 4.0$ & 25.5 & $10(7 / 3)$ & $63-74$ & 6 \\
\hline Johnson et $\mathrm{a}^{24}$ & $48(28 / 21)$ & $65.0 \pm 8.0$ & II-III & $\begin{array}{l}4.6 \pm 0.6 \\
7.2 \pm 0.9 \\
\end{array}$ & $\begin{array}{l}12.7 \pm 1.7 \\
17.5 \pm 1.9\end{array}$ & $17(10 / 7)$ & $64 \pm 7$ & 7 \\
\hline $\begin{array}{l}\text { Zawadka- } \\
\text { Kunikowska et al }\left.\right|^{36}\end{array}$ & I5 (NR) & $73.5 \pm 9.7$ & I-III & NR & NR & 24 (NR) & $42-90$ & 5 \\
\hline Fukunaga et $\mathrm{al}^{43}$ & $30(12 / 18)$ & $59.8 \pm 10.3$ & I-III & NR & NR & $29(I 8 / I I)$ & $58.9 \pm 9.8$ & 6 \\
\hline Oude Nijhuis et $\mathrm{al}^{28}$ & $7(1 / 6)$ & $56.1 \pm 68.6$ & II-III & $5.0 \pm 1.8$ & $41.0 \pm 17.1$ & $8(1 / 7)$ & $53.4 \pm 67.0$ & 6 \\
\hline Ferrazzoli et a $\left.\right|^{26}$ & $29(17 / 12)$ & $69.2 \pm 8.8$ & II-III & $10.6 \pm 5.2$ & $18.6 \pm 5.5$ & $12(9 / 3)$ & $66.5 \pm 6.3$ & 6 \\
\hline Fernandes et $\mathrm{a}^{37}$ & 50 (NR) & $68.3 \pm 7.3$ & II & NR & $19.1 \pm 7.9$ & 60 (NR) & $68.9 \pm 10.1$ & 6 \\
\hline Barbosa et $\mathrm{al}^{25}$ & $40(16 / 24)$ & $67.2 \pm 4.3$ & II-III & NR & NR & $27(17 / 10)$ & $68.37 \pm 3.7$ & 6 \\
\hline Barbieri et al ${ }^{32}$ & 19 (NR) & $72.7 \pm 5.9$ & I-III & NR & $\begin{array}{l}13.6 \pm 2.8 \\
27.5 \pm 7.7\end{array}$ & II (5/6) & $69.18 \pm 7.37$ & 6 \\
\hline Doná et a ${ }^{40}$ & $4 \mid(12 / 29)$ & $40-80$ & I-III & $8.0 \pm 6.0$ & $27.0 \pm I 4.0$ & $4 I(19 / 22)$ & $62 \pm 12$ & 6 \\
\hline Rocchi et $\mathrm{al}^{44}$ & $21(5 / 16)$ & $61.7 \pm 7.8$ & II-III & $16.2 \pm 9.2$ & $22.4 \pm 11.2$ & $24(6 / 18)$ & $62.4 \pm 7.4$ & 5 \\
\hline Lewek et $\mathrm{a}^{23}$ & $12(9 / 3)$ & $68.0 \pm 8.0$ & 1 & $2.0 \pm 0.8$ & $11.2 \pm 5.5$ & $8(3 / 5)$ & $61 \pm 12$ & 6 \\
\hline Vitório et al ${ }^{50}$ & $12(4 / 8)$ & $67.0 \pm 6.2$ & I-III & $7.1 \pm 5.5$ & $30.9 \pm 19.0$ & $12(4 / 8)$ & $67 \pm 6.44$ & 6 \\
\hline Brown et $\mathrm{al}^{46}$ & $10(3 / 7)$ & $67.2 \pm 6.1$ & II-III & $4.2 \pm 7.3$ & $27.3 \pm 5.3$ & $10(7 / 3)$ & $65.9 \pm 6.0$ & 6 \\
\hline Rogers et al $4^{45}$ & $8(3 / 5)$ & $72.9 \pm 9.3$ & II & $4.3 \pm 3.2$ & $16.8 \pm 4.5$ & $8(3 / 5)$ & $73.3 \pm 9.1$ & 6 \\
\hline Vitório et al ${ }^{49}$ & $30(16 / 14)$ & $70.8 \pm 6.9$ & I-III & NR & $\begin{array}{l}18.2 \pm 5.6 \\
28.9 \pm 6.9\end{array}$ & I5 (8/7) & $70.7 \pm 5.1$ & 6 \\
\hline Doan et a ${ }^{47}$ & $10(5 / 5)$ & $69.7 \pm 10.3$ & NR & $8.2 \pm 6.6$ & $18.1 \pm 10.5$ & 10 (NR) & $68.8 \pm 8.4$ & 5 \\
\hline Galna et al ${ }^{48}$ & $20(7 / / 3)$ & $65.6 \pm 7.7$ & I-III & NR & $12.6 \pm 5.1$ & $20(7 / / 3)$ & $65.3 \pm 8.0$ & 6 \\
\hline
\end{tabular}

Abbreviations: F, female; H\&Y, Hoehn and Yahr; M, male; NR, not reported; PD, Parkinson's disease; PEDro, Physiotherapy Evidence Database; UPDRS, Unified Parkinson's Disease Rating Scale.

which has been developed to assess the disability of patients and has become the gold standard in the clinical judgment of PD patients. The scale consists of four main elements: the state of intellectual and mood disorders (Part I), activities of daily life (Part II), motor symptoms and balance disorders
(Part III), and complications of treatment (Part IV). The UPDRS is predominantly used in clinical settings and in research on Parkinson's disease. ${ }^{22}$

The most conventional clinical tests to assess the balance in PD are the Berg Balance Scale (BBS); ${ }^{23-26}$ Timed Up and 
Go (TUG) Test; ${ }^{24}$ Tinetti test; ${ }^{27,28}$ and pull-test, tandem walk, and single-leg standing tests. ${ }^{11,18}$

\section{Objective assessment of balance}

Beyond all these clinical assessments, there is computerized posturography, which has been widely used to quantify the postural control by analyzing the displacement of the center of foot pressure (COP). In our review, almost all the authors used different types of force platforms. Most of the authors assessed static balance on stable platforms: AMTI AccuGait, AccuSway, ${ }^{24,25,29-32}$ QFP Medicapture platform, ${ }^{15,27,33}$ GK-1000, ${ }^{34}$ SATEL platform,,${ }^{35}$ and PROMED. ${ }^{36}$ In some articles, the following pressure platforms were used: T\&T Medilogic $\mathrm{GmbH}^{17}$ and Emed AT25; ${ }^{37}$ in other articles, platforms were connected to monitors and virtual reality was used: Bertec 4060-NC, ${ }^{38}$ the BRUTM Balance Rehabilitation Unit, ${ }^{39,40}$ and the Smart Balance Master System. ${ }^{41}$ Researchers sometimes applied a system designed to test balance and stability that provided balance training for patients; the system used was a mobile platform: the Biodex Balance System, ${ }^{42}$ Tetrax System, ${ }^{43}$ Prokin $254,{ }^{26}$ and a dual-axis platform. ${ }^{28}$

In addition to the objective assessment of static balance, there were many scientific articles that evaluated dynamic balance in PD. The authors mainly examined step initiation ${ }^{44,45}$ and gait with ${ }^{46-49}$ or without crossing an obstacle..$^{23}$ In most cases, the authors used an optoelectronic three-dimensional system with a walkway, ${ }^{23,46,49}$ sometimes these systems were used together with a force platform, ${ }^{45,48}$ but sometimes the force platform was used only with a walkway. ${ }^{44,47}$

\section{Posturographic measurement procedures used in PD}

Postural instability is one of the most common problems for people with $\mathrm{PD}$ and is extensively diagnosed by force plate posturography. In reviewing the literature, we noticed that there were many different measurement procedures. The most standard procedures were the quiet stance (QS) test $(n=20)$ and the limits of stability (LOS) test $(n=7)$. In most cases, the duration of barefoot standing was 30 seconds $(n=7)$, 32 seconds $(n=2)$, and 60 seconds $(n=6)$, and in one case, the trial lasted for 10 seconds. However, there was also a test in which the trial lasted for 51.2 or 25.6 seconds. ${ }^{27,35}$ The duration of the single-leg stance test was 20 seconds, ${ }^{51}$ and the duration of the LOS test was 20 seconds $^{41,42}$ or 15 seconds. ${ }^{52}$ Each trial was repeated one time or two to eight times, but the trial was mostly repeated three times. Postural instability was also measured at several positions of the foot: double leg $(n=17)$, single leg $(n=2)$, and tandem stance $(n=2)$. The detailed characteristics of the measurement procedures and conditions are shown in Table 3.
The ability to maintain balance depends on the integration of the proprioceptive, vestibular, and visual systems. ${ }^{53}$ Therefore, the researchers assessed the balance under various conditions. Static balance was measured with the eyes open and closed $(n=20)$ and on both stable and unstable surfaces. In some articles, the authors investigated standing balance in individuals with PD under single- and dual-task conditions, such as counting or talking $25,26,37,38$ and with the support of surface perturbation..$^{28}$

Impaired balance control is also associated with gait disturbance in PD patients. Comparing to standing the gait is a big challenge for maintaining balance, and it is well known that the postural instability increases dramatically as the BOS changes from a double-leg stance to a singleleg stance during a step. ${ }^{54}$ Patients with PD exhibit a deficit in maintaining balance during transition between states of static and dynamic equilibrium, such as gait initiation. PD can perform straight line walking task relatively easily, but they experience severe problems during more demanding tasks such as crossing obstacles..$^{55}$ Therefore, in this review, we included studies that consider the most demanding gait constrains in PD.

The researchers used different procedures to assess these aspects of dynamic balance; two of them investigated only gait initiation. ${ }^{44,45}$ Rocchi et al ${ }^{44}$ tested step initiation when the initial stance changed from narrow to wide. In this study, the subjects were instructed to take two steps, starting with the right foot at their comfortable rate and step initiation trials were performed three times, whereas Rogers et $\mathrm{a}^{45}$ examined the effect of perturbations of ground support on postural control during step initiation in PD.

Some authors ${ }^{48,49}$ examined gait along a walkway at their preferred speed and crossing an obstacle. Vitório et $\mathrm{al}^{49}$ measured obstacle avoidance three times, and the participants were also informed to step over the obstacle with their right leg. Galna et a ${ }^{48}$ tested four trials for levelground walking and eight trials for walking with obstacle avoidance. In another study, Brown et $\mathrm{a}^{46}$ explored whether a concurrent task affected obstacle crossing. In their study, subjects walked along a walkway at a self-determined pace under six different testing conditions: music accompaniment (no music/music), a concurrent arithmetic task (single task/ dual task), and walking without or with crossing an obstacle. In our review, we also found that some authors recorded gait under three conditions: preferred gait velocity, fastest possible gait velocity and walking on the heels. All of these trials were performed five times. ${ }^{23}$

Furthermore, in Doan et al, ${ }^{47}$ the researchers tested patients with PD during stepping over an obstacle in a threatening 
context. Participants walked under two separate conditions: on the gait path with the obstacle raised above the floor and on the gait path with the obstacle at floor level. Each trial was repeated 18 times. The detailed characteristics of the measurements procedures and conditions are shown in Table 1 .

\section{Postural instability in PD: results of objective methods}

\section{Static balance}

Many researchers have investigated the postural instability in patients with PD. Postural sway can be measured by a force platform that registers displacements of the COP. Alterations in body sway can be described by numerous variables, such as the area, range, velocity, frequency, path length, root mean square, and SD of the COP. ${ }^{56}$ All these COP measures and the range of the LOS were estimated in the reviewed articles.

The searched literature that performed an objective posturographic assessment provided contradictory results. A large majority of the studies revealed that PD patients exhibited body sway at higher rates than that of healthy agedmatched subjects $(n=12)$, whereas other findings showed similar $(n=4)$ and smaller $(n=2)$ body sway.

According to some researchers, ${ }^{15,25,30,33-35,40}$ patients with PD, during a QS both with eyes open and eyes close, presented a significantly greater spontaneous sway area than elderly people without neurodegenerative problems. In addition, the total anteroposterior (AP) and mediolateral (ML) sway path lengths were higher in PD. 15,30,33,35 Also, the mean velocity of the COP oscillation was significantly different between PD patients and the aged-matched control groups. ${ }^{25,34}$ On the other hand, in some articles, analysis of the COP area and path COP (length and velocity) during QS showed a lack of significant differences between $P D$ patients and elderly people without neurodegenerative problems. ${ }^{24,27,36}$ Moreover, the mean values of the AP and ML sway range measured in the PD group were significantly higher compared to those of the control group. ${ }^{15,33,34,37}$ Similarly, in other studies, the SDs of the COP were significantly greater in PD patients. ${ }^{25,26}$ Furthermore, Ebersbach and Gunkel ${ }^{17}$ compared PD patients (with a normal pull-test and with pull-test score of 1 or 2) and healthy controls. In this study, they observed that patients with a normal pull-test had lower sway than the control group in static and dynamic posturography. Termoz et $\mathrm{al}^{29}$ compared the postural control mechanisms of young, elderly, and PD patients. These authors suggested similar postural control mechanisms in young, elderly, and PD subjects. However, PD subjects showed significantly smaller root mean square amplitudes compared to the elderly people. These findings imply that PD patients use a stiffening strategy to control their balance.

The visual system yields central nervous system information regarding the position of the body relative to the environment. In the abovementioned studies, the authors found that all of the recorded variables increased after visual deprivation.

Another very important aspect is falling, which is very common in PD patients who has a high risk of falling. ${ }^{43}$ In comparison to the control group, PD patients showed significantly less confidence in maintaining their balance and preventing falls during the performance of daily activities, which was associated with the increasing area of the COP. ${ }^{51}$ In addition, Johnson et $\mathrm{al}^{24}$ used posturography to assess postural stability and discriminate between fallers and nonfallers in PD. Their results indicated that the static posturography variables measured allowed discrimination of fallers from elderly people without neurodegenerative problems, while none of these variables discriminated fallers from nonfallers. Regarding dynamic balance, fallers exhibited significantly slower reaction times than nonfallers and controls and also had a significant slowing of their movement velocity compared to nonfallers and controls.

Many studies found effects of cognitive tasks and a simple motor task in static balance disturbance in PD. ${ }^{25-27,37,38}$ Some authors $^{26,27}$ found that the COP area and the SD of the COP were significantly increased in PD patients during dual task performance, whereas no difference of the COP path was observed. Similarly, Barbosa et $\mathrm{al}^{25}$ showed that balance in the dual task was significantly poorer than balance with eyes closed. However, Fernandes et $\mathrm{al}^{37}$ and Schmit et $\mathrm{al}^{38}$ found that the influence of performing an additional task on COP displacement was similar for both the PD and elderly groups.

Under clinical conditions, balance disorders are diagnosed with a score of stage 3 or higher on the H\&Y scale. In our review, we noticed that some studies examined the PD population in the early stages of $\mathrm{PD}^{30,35,37}$ and compared them to those with moderate and high disease severity. ${ }^{32}$ Stylianou et $\mathrm{al}^{30}$ claimed that ML sway path length, sway area, and sway range are sensitive to the effects of PD even in mild to moderate severity patients. Similarly, Chastan et $\mathrm{al}^{35}$ investigated sway path length and sway area, but they noticed significant difference between groups only in sway area. Also Fernandes et $\mathrm{al}^{37}$ noticed that the AP and ML sway range were significantly greater in early stage $\mathrm{PD}$ than control subjects. In addition, individuals with PD in H\&Y stage II-III 
presented higher postural control asymmetry than those in H\&Y stage I. In addition, PD in II-III also presented higher postural control asymmetry than neurologically healthy individuals. ${ }^{32}$ According to all these results, postural instability exists in the early stages of PD before clinical symptoms. Moreover, Błaszczyk et $\mathrm{al}^{15}$ and Błaszczyk and Orawiec ${ }^{33}$ performed a significant correlation between $\mathrm{H} \& Y$ stage and sway range under both eyes open and closed conditions and ML sway path length under eyes open condition. H\&Y stages are also associated with risk of falls.

Most of reviewed studies reported disease duration, but not everyone correlate it with results. Błaszczyk and Orawiec $^{33}$ found that in the PD patient, both AP and ML sway ranges recorded with eyes closed correlated with the duration of the disease. Also the ML sway ratio correlated with PD duration. However, Johnson et $\mathrm{al}^{24}$ revealed no correlation between disease duration and static and dynamic posturography.

Many authors investigated the LOS in different directions. ${ }^{31,39-42,52,57}$ The LOS values were significantly lower (suggesting impaired balance and increased risk of falling) in PD patients compared to controls. ${ }^{40,42,52}$ PD subjects had smaller postural stability margins in all directions than elderly people, but PD patients maintained greater stability in the forward direction compared to the backward direction. ${ }^{40,56}$ Moreover, Bonnet et $\mathrm{al}^{31}$ tested impairments in ML body shifts. In this study, PD patients showed a significantly lower contribution of the ML postural control mechanisms compared with elderly control subjects. In addition, Vervoort et $\mathrm{al}^{41}$ analyzed the ability to move the COP ML and AP in a group of PD patients (freezers and nonfreezers) and age-matched controls. This study revealed that freezers performed poorer in a directional postural control task compared to nonfreezers and control subjects.

Some authors correlated posturographic and clinical variables. $^{24-27,33}$ The authors most commonly investigated relationships between posturography and BBS, TUG test, Tinetti test, and UPDRS. Johnson et $\mathrm{al}^{24}$ noticed the strong correlations with the BBS and TUG time, which were linearly related to falls and which were significantly positive correlated with the sway area. However, in some studies, there was only a weak correlation between ML SD of the COP and BBS in eyes open condition. ${ }^{25}$ Also, Ferrazzoli et $\mathrm{al}^{26}$ show a significant negative correlation between BBS scores and ML $\mathrm{SD}$ of the COP and sway area. But the same authors pointed no significant correlation between posturographic parameters and disease severity evaluated with UPDRS. Similarly, Marchese et $\mathrm{al}^{27}$ presented no significant correlation among
UPDRS, Tinetti test, and mean values of COP area and COP path under both visual conditions. But in other study, there was a significant positive correlation between the ML sway ratio under eyes open conditions and the UPDRS. ${ }^{33}$

\section{Dynamic balance during gait initiation and walking}

Gait initiation is a motor task that involves alternating from a QS in double-limb support to a dynamic balance that allows forward body movement; gait initiation consists of a preparatory phase and stepping phase. ${ }^{58}$ The preparatory phase is associated with anticipatory postural adjustments (APAs) in which the COP shifts backward and toward the swing limb to move the body center of mass (COM) forward and over the stance limb prior to stepping. ${ }^{58}$ Some studies have indicated that in comparison with healthy control subjects, APA in PD patients may be abnormal. In the patients with PD, self-initiated steps were characterized by a longer APA duration, longer time to first step onset, and slower step speed than those of controls. ${ }^{45}$ Sometimes, APAs were tested during step initiation from a narrow-stance and wide-stance BOS. The results indicated that the preparation for gait initiation from a wide stance was associated with a larger lateral and backward COP displacement than that from a narrow stance, and PD patients had much more difficulty starting to step from a wide stance. ${ }^{44}$

Gait in PD is a topic of growing interest for researchers. Many authors examined straight line walking and more demanding tasks for PD patients, such as crossing an obstacle. Lewek et $\mathrm{al}^{23}$ performed an examination of arm swing magnitude and asymmetry in individuals with early PD, and their results indicated that there were no significant differences in arm swing magnitude between the PD and control groups. Considering obstacle avoidance, subjects with PD crossed the obstacle slower than healthy subjects. ${ }^{46,47}$ Some people with PD walked with greater and faster ML sway than control subjects, particularly when walking over obstacles. In addition, PD patients maintained their COM more medial to their COP compared to the control group when transferring the lead limb over the obstacle. These results suggest that $\mathrm{PD}$ reduces the risk of lateral falls. ${ }^{48}$

\section{Discussion}

A systematic review was conducted on the different methods and various posturographic procedures and standard measures of postural sway variability used in studies on balance disorders in individuals with PD. This analysis identified many potentially important differences in the included studies. 
The postural changes in PD are widely described by different authors, but the results are often dissimilar and contradictory. Some reviewed articles showed that patients with PD have higher values of the COP variables than people without neurological deficits..$^{15,30,33-35,38}$ However, other studies shown that COP variables are lower in patients with PD than in healthy subjects, ${ }^{17,29}$ and Zawadka-Kunikowska et $\mathrm{al}^{36}$ noted no significant differences between groups, and also Fernandes et $\mathrm{al}^{37}$ and Marchese et $\mathrm{al}^{27}$ obtained similar results. This might be due to the sample size. Although seven articles had fewer than 15 participants in each of their groups, ${ }^{28,29,34,35,38,52,57}$ out of the 24 studies focused on static balance included in this review, none reported the sample size calculation results. In addition, some studies have not equinumerous patients and control groups. ${ }^{17,24,25,27,36}$ Although it is important to emphasize that a large sample size is not always required, reporting the outcome of an appropriate statistical power calculation could be beneficial for reported findings.

Furthermore, postural difficulties are different during PD progression and at each stage of $\mathrm{PD}$. According to the $\mathrm{H} \& \mathrm{Y}$ scale, the balance disorders occur only in the third stage. ${ }^{10}$ However, it has been reported that postural instability may appear already in the early stages of the disease, even before the onset of clinical symptoms. ${ }^{11}$ The current knowledge about changes in postural control in mild to moderate PD is limited and unclear, but it is known that the early stage PD patients had a larger sway area, ${ }^{35} \mathrm{COP}$ displacement, and COP velocity ${ }^{37}$ than the control subjects. This systematic review indicated that there are only few articles that consider balance dysfunction in early stages of the disease, and the majority of authors assess postural control in the entire PD population (H\&Y stage I-III) without dividing into mild to moderate stages. These issues can negatively affect the future interpretations of results and give contradictory information about postural control in people with PD.

Another issue that was noticed in the review is different duration of QS and various numbers of repetitions of each trial. It is known that in most cases of common COP measures, it will be enough to average three 30 seconds trials or one to two 60 seconds trials to achieve acceptable reliability $(\mathrm{R}=0.7) .{ }^{59}$ Within the included studies, we have noticed that some authors employed different measurements procedures. Termoz et $\mathrm{al}^{29}$ applied five 10 seconds trials; other authors used one to two 32 seconds trials, ${ }^{36,43} 8$ of 51 2 seconds trials, ${ }^{27}$ or one to two 30 seconds trials; ${ }^{34,38}$ and two studies did not report information about duration and repetitions of trials..$^{39,40}$ These are articles where we noticed different results compared to other studies. Moreover, what is also significant in research planning, another potential reason regarding discrepancy in the results is that the authors use diverse equipment and sampling frequency leading to different outcome measures. Of the 24 reviewed studies, 14 articles reported sampling frequency that ranged from 10 to $200 \mathrm{~Hz}$.

Although it was not the primary goal of our review to evaluate the effects of PD-related parameters (eg, UPDRS), disease duration or antiparkinsonian medications on measures of standing balance and walking stability, they are important factors that should be considered. It is widely recognized that levodopa reduced rigidity and improved bradykinesia and gait speed, ${ }^{60}$ but it have little impact on balance. ${ }^{61}$ However, in most included studies, the PD patients were tested in the "ON" state after the intake of dopaminergic drugs. Only one of the reviewed articles investigated the levodopa effect on postural control. We also noticed that most studies reported disease duration, but the authors of only two articles compared this aspect with their results. ${ }^{24,33}$ Therefore, there is a clear need for further research in this area.

\section{Conclusion}

PD is the most common neurodegenerative disease and has been widely investigated by many researchers. It is well known that PD patients have numerous balance and motor disorders, and investigators have examined many different aspects of these disabilities. In our review, we found that tests of both static and dynamic balances in PD were performed and that the authors used various subjective and objective methods. Regarding the objective methods, we found that there were many dissimilar procedures in posturography. There were various measurement durations, conditions, variables, and equipments used. In our opinion, to better assess postural control in PD, reliable procedures to measure balance should be established. Future researchers in this topic should precisely define the reliability and validity of the measures and methods, duration and number of repetitions of trials, sample size, and sampling frequency. Furthermore, future studies should take account of specific PD-related characteristics such as the stage of the disease. Thereby, it is possible to create a gold standard in posturography measures in PD. In addition, we suggest using objective methods (for example, force plate) to assess balance, with standard clinical tests as supplementary procedures. 


\section{Acknowledgment}

The study was supported by the National Center for Research and Development Grant under the program STRATEG MED III within the "VB-Clinic" project no STRATEGMED3/306011/1/NCBR/2017.

\section{Disclosure}

The authors report no conflicts of interest in this work.

\section{References}

1. Winter DA. Human balance and posture control during standing and walking. Gait Posture. 1995;3(4):193-214.

2. Zatsiorsky VM, Duarte M. Rambling and trembling in quiet standing. Motor Control. 2000;4(2):185-200.

3. Hof AL. The equations of motion for a standing human reveal three mechanisms for balance. J Biomech. 2007;40(2):451-457.

4. Benatru I, Vaugoyeau M, Azulay JP. Postural disorders in Parkinson's disease. Neurophysiol Clin. 2008;38(6):459-465.

5. Jankovic J. Parkinson's disease and movement disorders: moving forward. Lancet Neurol. 2008;7(1):9-11.

6. Opara J, Małecki A, Małecka E, Socha T. Motor assessment in Parkinson's disease. Ann Agric Environ Med. 2017;24(3):411-415.

7. Bloem BR, Beckley DJ, van Hilten BJ, Roos RA. Clinimetrics of postural instability in Parkinson's disease. J Neurol. 1998;245(10):669-673.

8. Salarian A, Horak FB, Zampieri C, Carlson-Kuhta P, Nutt JG, Aminian K. iTUG, a sensitive and reliable measure of mobility. IEEE Trans Neural Syst Rehabil Eng. 2010;18(3):303-310.

9. Hubble RP, Silburn PA, Naughton GA, Cole MH. Assessing stability in mild and moderate Parkinson's disease: Can clinical measures provide insight? Gait Posture. 2016;49:7-13.

10. Hoehn MM, Yahr MD. Parkinsonism: onset, progression, and mortality Parkinsonism: onset, progression, and mortality. Neurology. 1967;17(5): 427-442.

11. Beuter A, Hernández R, Rigal R, Modolo J, Blanchet PJ. Postural sway and effect of levodopa in early Parkinson's disease. Can J Neurol Sci. 2008;35(1):65-68.

12. Nardone A, Schieppati M. Balance in Parkinson's disease under static and dynamic conditions. Mov Disord. 2006;21(9):1515-1520.

13. Visser JE, Carpenter MG, van der Kooij H, Bloem BR. The clinical utility of posturography. Clin Neurophysiol. 2008;119(11):2424-2436.

14. Snijders AH, Leunissen I, Bakker M, et al. Gait-related cerebral alterations in patients with Parkinson's disease with freezing of gait. Brain. 2011;134(Pt 1):59-72.

15. Błaszczyk JW, Orawiec R, Duda-Kłodowska D, Opala G. Assessment of postural instability in patients with Parkinson's disease. Exp Brain Res. 2007;183(1):107-114.

16. Matinolli M, Korpelainen JT, Korpelainen R, Sotaniemi KA, Virranniemi M, Myllylä VV. Postural sway and falls in Parkinson's disease: a regression approach. Mov Disord. 2007;22(13):1927-1935.

17. Ebersbach G, Gunkel M. Posturography reflects clinical imbalance in Parkinson's disease. Mov Disord. 2011;26(2):241-246.

18. Bhandari M, Giannoudis PV. Evidence-based medicine: what it is and what it is not. Injury. 2006;37(4):302-306.

19. Sharififar S, Coronado RA, Romero S, Azari H, Thigpen M. The effects of whole body vibration on mobility and balance in Parkinson disease: a systematic review. Iran J Med Sci. 2014;39(4):318-326.

20. Winser SJ, Tsang WW, Krishnamurthy K, Kannan P. Does Tai Chi improve balance and reduce falls incidence in neurological disorders? A systematic review and meta-analysis. Clin Rehabil. 2018; 32(9):1157-1168.

21. Martínez-Martín P, Gil-Nagel A, Gracia LM, Gómez JB, MartínezSarriés J, Bermejo F. Unified Parkinson's disease rating scale characteristics and structure. Mov Disord. 1994;9(1):76-83.
22. Ebersbach G, Baas H, Csoti I, Müngersdorf M, Deuschl G. Scales in Parkinson's disease. J Neurol. 2006;253(Suppl 4):iv32-iv35.

23. Lewek MD, Poole R, Johnson J, Halawa O, Huang X. Arm swing magnitude and asymmetry during gait in the early stages of Parkinson's disease. Gait Posture. 2010;31(2):256-260.

24. Johnson L, James I, Rodrigues J, Stell R, Thickbroom G, Mastaglia F. Clinical and posturographic correlates of falling in Parkinson's disease. Mov Disord. 2013;28(9):1250-1256.

25. Barbosa AF, Souza C de O, Chen J, et al. The competition with a concurrent cognitive task affects posturographic measures in patients with Parkinson disease. Arq Neuropsiquiatr. 2015;73(11):906-912.

26. Ferrazzoli D, Fasano A, Maestri R, et al. Balance Dysfunction in Parkinson's Disease: The Role of Posturography in Developing a Rehabilitation Program. Parkinsons Dis. 2015;2015:520128.

27. Marchese R, Bove M, Abbruzzese G. Effect of cognitive and motor tasks on postural stability in Parkinson's disease: a posturographic study. Mov Disord. 2003;18(6):652-658.

28. Oude Nijhuis LB, Allum JH, Nanhoe-Mahabier W, Bloem BR. Influence of perturbation velocity on balance control in Parkinson's disease. PLoS One. 2014;9(1):e86650-e86657.

29. Termoz N, Halliday SE, Winter DA, Frank JS, Patla AE, Prince F. The control of upright stance in young, elderly and persons with Parkinson's disease. Gait Posture. 2008;27(3):463-470.

30. Stylianou AP, McVey MA, Lyons KE, Pahwa R, Luchies CW. Postural sway in patients with mild to moderate Parkinson's disease. Int $J$ Neurosci. 2011;121(11):614-621.

31. Bonnet CT, Delval A, Defebvre L. Interest of active posturography to detect age-related and early Parkinson's disease-related impairments in mediolateral postural control. J Neurophysiol. 2014;112(10):2638-2646.

32. Barbieri FA, Polastri PF, Baptista AM, et al. Effects of disease severity and medication state on postural control asymmetry during challenging postural tasks in individuals with Parkinson's disease. Hum Mov Sci. 2016;46:96-103.

33. Błaszczyk JW, Orawiec R. Assessment of postural control in patients with Parkinson's disease: sway ratio analysis. Hum Mov Sci. 2011;30(2):396-404.

34. Ickenstein GW, Ambach H, Klöditz A, et al. Static posturography in aging and Parkinson's disease. Front Aging Neurosci. 2012;4:20.

35. Chastan N, Debono B, Maltête D, Weber J. Discordance between measured postural instability and absence of clinical symptoms in Parkinson's disease patients in the early stages of the disease. Mov Disord. 2008;23(3):366-372.

36. Zawadka-Kunikowska M, Zalewski P, Klawe JJ, et al. Age-related changes in cognitive function and postural control in Parkinson's disease. Aging Clin Exp Res. 2014;26(5):505-510.

37. Fernandes Â, Coelho T, Vitória A, et al. Standing balance in individuals with Parkinson's disease during single and dual-task conditions. Gait Posture. 2015;42(3):323-328.

38. Schmit JM, Riley MA, Dalvi A, et al. Deterministic center of pressure patterns characterize postural instability in Parkinson's disease. Exp Brain Res. 2006;168(3):357-367.

39. Suarez H, Geisinger D, Ferreira ED, et al. Balance in Parkinson's disease patients changing the visual input. Braz J Otorhinolaryngol. 2011;77(5): 651-655.

40. Doná F, Aquino CC, Gazzola JM, et al. Changes in postural control in patients with Parkinson's disease: a posturographic study. Physiotherapy. 2016;102(3):272-279.

41. Vervoort G, Nackaerts E, Mohammadi F. Which Aspects of Postural Control Differentiate Between Freezing and Non-Freezing Patients With Parkinson'S Disease? Parkinsonism Relat Disord. 2013;2013:971480.

42. Ganesan M, Pal PK, Gupta A, Sathyaprabha TN. Dynamic posturography in evaluation of balance in patients of Parkinson's disease with normal pull test: concept of a diagonal pull test. Parkinsonism Relat Disord. 2010;16(9):595-599.

43. Fukunaga JY, Quitschal RM, Doná F, Ferraz HB, Ganança MM, Caovilla HH. Postural control in Parkinson's disease. Braz J Otorhinolaryngol. 2014;80(6):508-514. 
44. Rocchi L, Chiari L, Mancini M, Carlson-Kuhta P, Gross A, Horak FB. Step initiation in Parkinson's disease: influence of initial stance conditions. Neurosci Lett. 2006;406(1-2):128-132.

45. Rogers MW, Hilliard MJ, Martinez KM, Zhang Y, Simuni T, Mille ML. Perturbations of ground support alter posture and locomotion coupling during step initiation in Parkinson's disease. Exp Brain Res. 2011; 208(4):557-567.

46. Brown LA, de Bruin N, Doan J, Suchowersky O, Hu B. Obstacle crossing among people with Parkinson disease is influenced by concurrent music. J Rehabil Res Dev. 2010;47(3):225-231.

47. Doan JB, de Bruin N, Pellis SM, Suchowersky O, Whishaw IQ, Brown LA. Obstacle Avoidance amongst Parkinson Disease Patients Is Challenged in a Threatening Context. J Neurodegener Dis. Epub 2013 May 15.

48. Galna B, Murphy AT, Morris ME. Obstacle crossing in Parkinson's disease: mediolateral sway of the centre of mass during level-ground walking and obstacle crossing. Gait Posture. 2013;38(4):790-794.

49. Vitório R, Lirani-Silva E, Baptista AM, et al. Disease severity affects obstacle crossing in people with Parkinson's disease. Gait Posture. 2014; 40(1):266-269.

50. Vitório R, Pieruccini-Faria F, Stella F, Gobbi S, Gobbi LT. Effects of obstacle height on obstacle crossing in mild Parkinson's disease. Gait Posture. 2010;31(1):143-146.

51. Adkin AL, Frank JS, Jog MS. Fear of falling and postural control in Parkinson's disease. Mov Disord. 2003;18(5):496-502.

52. Mancini M, Rocchi L, Horak FB, Chiari L. Effects of Parkinson's disease and levodopa on functional limits of stability. Clin Biomech (Bristol, Avon). 2008;23(4):450-458.
53. Horak FB. Postural orientation and equilibrium: what do we need to know about neural control of balance to prevent falls? Age Ageing. 2006;35(Suppl 2):ii7-ii11.

54. Winter DA, Patla AE, Frank JS. Assessment of balance control in humans. Med Prog Technol. 1990;16(1-2):31-51.

55. Morris ME, Huxham F, McGinley J, Dodd K, Iansek R. The biomechanics and motor control of gait in Parkinson disease. Clin Biomech (Bristol, Avon). 2001;16(6):459-470.

56. Rocchi L, Chiari L, Cappello A, Horak FB. Identification of distinct characteristics of postural sway in Parkinson's disease: a feature selection procedure based on principal component analysis. Neurosci Lett. 2006; 394(2):140-145.

57. Horak FB, Dimitrova D, Nutt JG. Direction-specific postural instability in subjects with Parkinson's disease. Exp Neurol. 2005;193(2): 504-521.

58. Massion J. Movement, posture and equilibrium: interaction and coordination. Prog Neurobiol. 1992;38(1):35-56.

59. Słomka K, Juras G, Sobota G, Bacik B. The reliability of a ramblingtrembling analysis of center of pressure measures. Gait Posture. 2013; 37(2):210-213.

60. Contin M, Riva R, Baruzzi A, Albani F, Macri' S, Martinelli P. Postural stability in Parkinson's disease: the effects of disease severity and acute levodopa dosing. Parkinsonism Relat Disord. 1996;2(1):29-33.

61. Horak FB, Frank J, Nutt J. Effects of dopamine on postural control in parkinsonian subjects: scaling, set, and tone. J Neurophysiol. 1996;75(6): 2380-2396
Clinical Interventions in Aging

\section{Publish your work in this journal}

Clinical Interventions in Aging is an international, peer-reviewed journal focusing on evidence-based reports on the value or lack thereof of treatments intended to prevent or delay the onset of maladaptive correlates of aging in human beings. This journal is indexed on PubMed Central, MedLine,

\section{Dovepress}

CAS, Scopus and the Elsevier Bibliographic databases. The manuscript management system is completely online and includes a very quick and fair peer-review system, which is all easy to use. Visit http://www.dovepress. com/testimonials.php to read real quotes from published authors. 